\title{
Exploring the origin of magnetic fields in massive stars
}

\section{New magnetic field measurements in cluster and field stars ${ }^{\star}$}

\author{
S. Hubrig ${ }^{1}$, M. Schöller ${ }^{2}$, I. Ilyin ${ }^{1}$, N. V. Kharchenko ${ }^{3}$, L. M. Oskinova ${ }^{4}$, N. Langer ${ }^{5}$, J. F. González ${ }^{6}$, A. F. Kholtygin ${ }^{7}$, \\ M. Briquet ${ }^{8, \star \star}$, and the MAGORI collaboration
}

\author{
${ }^{1}$ Leibniz-Institut für Astrophysik Potsdam (AIP), An der Sternwarte 16, 14482 Potsdam, Germany \\ e-mail: shubrig@aip.de \\ 2 European Southern Observatory, Karl-Schwarzschild-Str. 2, 85748 Garching, Germany \\ 3 Main Astronomical Observatory, 27 Academica Zabolotnogo Str., 03680 Kiev, Ukraine \\ ${ }^{4}$ Universität Potsdam, Institut für Physik und Astronomie, 14476 Potsdam, Germany \\ 5 Argelander-Institut für Astronomie, Universität Bonn, Auf dem Hügel 71, 53121 Bonn, Germany \\ ${ }^{6}$ Instituto de Ciencias Astronomicas, de la Tierra, y del Espacio (ICATE), 5400 San Juan, Argentina \\ 7 Astronomical Institute, Saint-Petersburg State University, Saint-Petersburg, Russia \\ 8 Institut d'Astrophysique et de Géophysique, Université de Liège, Allée du 6 Août 17, 4000 Liège, Belgium
}

Received 12 November 2012 / Accepted 11 January 2013

\section{ABSTRACT}

\begin{abstract}
Context. Theories on the origin of magnetic fields in massive stars remain poorly developed, because the properties of their magnetic field as function of stellar parameters could not yet be investigated. Additional observations are of utmost importance to constrain the conditions that are conducive to magnetic fields and to determine first trends about their occurrence rate and field strength distribution. Aims. To investigate whether magnetic fields in massive stars are ubiquitous or appear only in stars with a specific spectral classification, certain ages, or in a special environment, we acquired 67 new spectropolarimetric observations for 30 massive stars. Among the observed sample, roughly one third of the stars are probable members of clusters at different ages, whereas the remaining stars are field stars not known to belong to any cluster or association.

Methods. Spectropolarimetric observations were obtained during four different nights using the low-resolution spectropolarimetric mode of FOcal Reducer low dispersion Spectrograph (FORS 2) mounted on the 8-m Antu telescope of the VLT. Furthermore, we present a number of follow-up observations carried out with the high-resolution spectropolarimeters SOFIN mounted at the Nordic Optical Telescope (NOT) and HARPS mounted at the ESO $3.6 \mathrm{~m}$ between 2008 and 2011. To assess the membership in open clusters and associations, we used astrometric catalogues with the highest quality kinematic and photometric data currently available.

Results. The presence of a magnetic field is confirmed in nine stars previously observed with FORS 1/2: HD 36879, HD 47839, CPD-28 2561, CPD-47 2963, HD 93843, HD 148937, HD 149757, HD 328856, and HD 164794. New magnetic field detections at a significance level of at least $3 \sigma$ were achieved in five stars: HD 92206c, HD 93521, HD 93632, CPD-468221, and HD 157857. Among the stars with a detected magnetic field, five stars belong to open clusters with high membership probability. According to previous kinematic studies, five magnetic O-type stars in our sample are candidate runaway stars.
\end{abstract}

Key words. polarization - stars: early-type - stars: kinematics and dynamics - stars: magnetic field - stars: massive open clusters and associations: general

\section{Introduction}

During the last years a gradually increasing number of $\mathrm{O}$, early B-type, and WR stars have been investigated for magnetic fields, and as a result, about a dozen magnetic O-type and two dozens of early B-type stars are presently known (e.g., Donati et al. 2006; Hubrig et al. 2008, 2011a,b; Martins et al. 2010; Wade et al. 2011, 2012a,b). The recent detections of magnetic fields in massive stars generate a strong motivation to study the correlations between evolutionary state, rotation velocity, and surface composition (Brott et al. 2011; Potter et al. 2012), and to understand the origin and the role of magnetic fields in massive stars (Langer 2012).

* Based on observations obtained at the European Southern Observatory, Paranal, Chile (ESO programmes 087.D-0049(A) and 187.D-0917(A)), and SOFIN observations at the $2.56 \mathrm{~m}$ Nordic Optical Telescope on La Palma.

$\star \star$ F.R.S.-FNRS Postdoctoral Researcher, Belgium.
The present study is the continuation of our magnetic surveys with FORS $1 / 2$ at the VLT of a sample of massive O and B-type stars in open clusters at different ages and in the field started already in 2005 (e.g., Hubrig et al. 2008, 2011b). In 2011 we presented the results of 41 spectropolarimetric FORS 2 observations of 36 massive stars. Among the observed sample, roughly half of the stars were probable members of clusters at different ages (Hubrig et al. 2011b). The new survey is based on four nights of observations with FORS 2 in 2011 May. Furthermore, we present a number of follow-up observations carried out with the high-resolution spectropolarimeters SOFIN mounted at the Nordic Optical Telescope (NOT) and HARPS mounted at the ESO $3.6 \mathrm{~m}$ between 2008 and 2011. The information on the occurrence rate of magnetic fields and field strength distribution is critical for answering the principal question of the possible origin of magnetic fields in massive stars.

In the following, we present 67 new measurements of magnetic fields in 30 massive stars. Our observations and the 
Table 1. List of massive stars observed for this programme.

\begin{tabular}{|c|c|c|c|}
\hline Name & $\begin{array}{c}\text { Other } \\
\text { identifier }\end{array}$ & V & Spectral type \\
\hline HD 36879 & $\mathrm{BD}+21899$ & 7.58 & O5 III (f) var \\
\hline HD 47839 & 15 Mon & 4.64 & O6.5 III (n)(f) \\
\hline CPD-28 2561 & CD-285104 & 10.09 & O6.5 f?p \\
\hline CPD-47 2963 & CD-474551 & 8.45 & O4 III (f) \\
\hline HD 77581 & Vela X-1 & 6.93 & B0.5 I ae* \\
\hline HD 92206c & CD-573378 & 9.07 & $\mathrm{O} 8.5 \mathrm{~V} \mathrm{p}$ \\
\hline HD 303225 & CD-593225 & 9.74 & $\mathrm{~B}^{*}$ \\
\hline HD 93026 & CD-583518 & 9.67 & B2 III* \\
\hline HD 93521 & $\mathrm{BD}+382179$ & 7.04 & $09.5 \mathrm{~V} \mathrm{p}$ \\
\hline HD 93632 & CD-593328 & 8.38 & O5 III (f) var \\
\hline CPD-58 2611 & CD-583526 & 9.55 & O6 V ((f)) \\
\hline HD 93843 & CD-55 5846 & 7.33 & O5 III (f) var \\
\hline HD 130298 & CD-55 5846 & 9.29 & O6.5 III (n)(f) \\
\hline HD 148937 & CD-47 10855 & 6.77 & O6.5 f?p \\
\hline HD 149757 & $\zeta \mathrm{Oph}$ & 2.58 & O9.5 V nn \\
\hline HD 328856 & CD-46 11016 & 8.50 & O9.5 III* \\
\hline CPD-468221 & CD-46 11017 & 9.19 & $\mathrm{O}+*$ \\
\hline HD 150958 & CD-46 11019 & 7.29 & O6.5 Ia (n)f + \\
\hline HD 151804 & HR 6245 & 5.25 & O8 Iaf \\
\hline HD 153426 & CD-38 11431 & 7.49 & O9 II-III \\
\hline HD 153919 & CD-37 11206 & 6.53 & O6.5 Ia f+ \\
\hline HD 154643 & CD-34 11503 & 7.15 & O9.5 III \\
\hline HD 156041 & CD-35 11432 & 9.2 & $\mathrm{~B} 0 \mathrm{~V}^{*}$ \\
\hline HD 156154 & CD-35 11445 & 8.04 & O8 Iab (f) \\
\hline HD 157857 & BD-104493 & 7.81 & O6.5 III (f) \\
\hline HD 164794 & 9Sgr & 5.93 & O4 V ((f)) \\
\hline HD 170580 & $\mathrm{BD}+033727$ & 6.69 & B2 $\mathrm{V}^{*}$ \\
\hline HD 170783 & $\mathrm{BD}+043778$ & 7.73 & B5* \\
\hline HD 172175 & BD-074642 & 9.44 & O6 I (n)f \\
\hline HD 191612 & $\mathrm{BD}+353995$ & 7.84 & O8 f?p \\
\hline
\end{tabular}

Notes. Spectral classifications are listed according to the Galactic O Star Catalogue (Maíz-Apellániz et al. 2004). All objects were observed with FORS 2, except HD 36879, HD 47839, and HD 191612, which were observed with SOFIN, HD 151804, which was observed with HARPS, and HD 164794, which was observed with HARPS and SOFIN. ${ }^{(*)}$ Indicates spectral types taken from SIMBAD.

obtained results are described in Sects. 2 and 3, and their discussion is presented in Sect. 4.

\section{Observations and results}

\subsection{FORS 2 observations}

The spectropolarimetric observations were carried out in 2011 May 4-8 in visitor mode at the European Southern Observatory (ESO) with FORS 2 mounted on the 8-m Antu telescope of the VLT. This multi-mode instrument is equipped with polarisation analysing optics, comprising super-achromatic halfwave and quarter-wave phase retarder plates, and a Wollaston prism with a beam divergence of $22^{\prime \prime}$ in standard resolution mode ${ }^{1}$. Polarimetric spectra were obtained with the GRISM 600B and the narrowest slit width of 0.4 to achieve a spectral resolving power of $R \sim 2000$. The use of the mosaic detector made of blue optimised E2V chips and a pixel size of $15 \mu \mathrm{m}$ allowed us to cover a large spectral range, from 3250 to $6215 \AA$, which includes all hydrogen Balmer lines from $\mathrm{H} \beta$ to the Balmer jump. The spectral types and the visual magnitudes of the studied stars are listed in Table 1.

\footnotetext{
1 The spectropolarimetric capabilities of FORS 1 were moved to FORS 2 in 2009.
}

A detailed description of the assessment of the longitudinal magnetic-field measurements using FORS 2 was presented in our previous papers (e.g., Hubrig et al. 2004a,b, and references therein). The mean longitudinal magnetic field, $\left\langle B_{z}\right\rangle$, was derived using

$$
\frac{V}{I}=-\frac{g_{\mathrm{eff}} e \lambda^{2}}{4 \pi m_{e} c^{2}} \frac{1}{I} \frac{\mathrm{d} I}{\mathrm{~d} \lambda}\left\langle B_{z}\right\rangle
$$

where $V$ is the Stokes parameter that measures the circular polarisation, $I$ is the intensity in the unpolarised spectrum, $g_{\mathrm{eff}}$ is the effective Landé factor, $e$ is the electron charge, $\lambda$ is the wavelength, $m_{\mathrm{e}}$ the electron mass, $c$ the speed of light, $\mathrm{d} I / \mathrm{d} \lambda$ is the derivative of Stokes $I$, and $\left\langle B_{z}\right\rangle$ is the mean longitudinal magnetic field. Errors are determined in the original spectra from photon statistics and propagated to the resulting $V / I$ spectra. The error of $\left\langle B_{z}\right\rangle$ is obtained from the formal error of the errorweighted least-squares fit.

Longitudinal magnetic fields were measured in two ways: using only the absorption hydrogen Balmer lines or the entire spectrum including all available absorption lines. The lines that show evidence of emission were not used to determine the magnetic field strength. In addition, a rectification was carried out for a few massive stars with continuum slopes detected in their Stokes $V$ spectra. The feasibility of longitudinal magnetic field measurements in massive stars using low-resolution spectropolarimetric observations has been demonstrated by previous studies of O and B-type stars (e.g., Hubrig et al. 2006, 2008, 2009a, 2011a,b).

Ten targets were observed once, 13 were observed two or three times. HD 148937 was observed four times to assess the magnetic field variability over the rotation cycle. Apart from this star, which has a rotation period of seven days (Nazé et al. 2010), no exact rotation periods are known for the other stars in our sample. The star $\zeta$ Oph was observed nine times with the aim to search for magnetic/rotational periodicity (see Sect. 3).

The results of our magnetic field measurements are presented in Table 2. In the first two columns, we provide the star names and the modified Julian dates at the middle of the exposures, followed by the signal-to-noise ratio $(\mathrm{S} / \mathrm{N})$ in the individual spectra in Col. 3. In Cols. 4 and 5, we present the longitudinal magnetic field $\left\langle B_{z}\right\rangle_{\text {all }}$ using the whole spectrum, and the longitudinal magnetic field $\left\langle B_{z}\right\rangle_{\text {hyd }}$ using only the hydrogen lines. All quoted errors are $1 \sigma$ uncertainties. In Col. 6 we indicate the wavelength regions used for the magnetic field measurements. The spectral regions containing the interstellar lines of the Ca II doublet are usually not used in the measurements. In Col. 7 we list the rotation phases for the Of?p star HD 148937, which is the only star in our FORS 2 observations with a known period of about seven days. Finally, in the last column we identify new detections by ND, previous detections by PD and confirmed detections by $\mathrm{CD}$.

In our sample of 25 stars observed with FORS 2 in 2011 May the presence of a magnetic field is confirmed in six stars previously observed with FORS 1/2 (Hubrig et al. 2008, 2011b,c): CPD-28 2561, CPD-47 2963, HD 93843, HD 148937, $\zeta$ Oph, and HD 328856. New magnetic field detections were achieved in five stars: HD 92206c, HD 93521, HD 93632, CPD-46 8221, and HD 157857. Although previous, mostly single observations of the five O-type stars HD 130298, HD 153426, HD 153919, HD 154643, and HD 156154 indicated the possible presence of weak magnetic fields (Hubrig et al. 2011b), our recent observations do not reveal magnetic fields at a significance level of $3 \sigma$ in these stars. We note that these recent observations have been 
S. Hubrig et al.: Exploring the origin of magnetic fields in massive stars. II.

Table 2. Longitudinal magnetic fields measured with FORS 2 in the studied sample.

\begin{tabular}{|c|c|c|c|c|c|c|c|}
\hline Name & MJD & $S / N$ & $\begin{array}{c}\left\langle B_{z}\right\rangle_{\text {all }} \\
{[\mathrm{G}]}\end{array}$ & $\begin{array}{c}\left\langle B_{z}\right\rangle_{\text {hydr }} \\
{[\mathrm{G}]}\end{array}$ & $\begin{array}{c}\text { Spectral } \\
\text { Regions }(\AA)\end{array}$ & Phase & Comment \\
\hline \multirow[t]{4}{*}{ CPD-282561 } & *55338.969 & 2262 & $-381 \pm 122$ & $-534 \pm 167$ & $3580-4588$ & & PD \\
\hline & 55685.982 & 2481 & $99 \pm 82$ & $65 \pm 108$ & & & \\
\hline & 55686.984 & 2516 & $-44 \pm 80$ & $6 \pm 102$ & & & \\
\hline & 55687.980 & 2490 & $269 \pm 81$ & $281 \pm 90$ & & & $\mathrm{CD}$ \\
\hline \multirow[t]{4}{*}{ CPD-47 2963} & *55337.094 & 2722 & $-190 \pm 62$ & $-154 \pm 96$ & $3696-5622$ & & PD \\
\hline & 55686.019 & 2691 & $-66 \pm 57$ & $-119 \pm 82$ & & & \\
\hline & 55687.037 & 3035 & $-169 \pm 52$ & $-77 \pm 76$ & & & CD \\
\hline & 55688.013 & 2164 & $95 \pm 69$ & $148 \pm 101$ & & & \\
\hline \multirow[t]{3}{*}{ Vela X-1 } & 55686.096 & 3604 & $-15 \pm 30$ & $-11 \pm 45$ & $3562-5886$ & & \\
\hline & 55687.058 & 2894 & $-80 \pm 32$ & $-114 \pm 50$ & & & \\
\hline & 55688.048 & 2433 & $57 \pm 37$ & $88 \pm 69$ & & & \\
\hline \multirow[t]{3}{*}{ HD 92206c } & 55686.135 & 2393 & $204 \pm 46$ & $140 \pm 88$ & $3658-5944$ & & ND \\
\hline & 55687.085 & 3439 & $-104 \pm 30$ & $-123 \pm 54$ & & & \\
\hline & 55688.151 & 2528 & $10 \pm 43$ & $23 \pm 46$ & & & \\
\hline HD 303225 & 55688.220 & 1902 & $-59 \pm 54$ & $-81 \pm 90$ & $3447-5299$ & & \\
\hline HD 93026 & 55687.247 & 1647 & $-19 \pm 75$ & $-52 \pm 100$ & $3470-4900$ & & \\
\hline \multirow[t]{3}{*}{ HD 93521} & 55686.042 & 3891 & $32 \pm 52$ & $45 \pm 73$ & $3689-5414$ & & \\
\hline & 55687.015 & 3304 & $-202 \pm 66$ & $-293 \pm 91$ & & & ND \\
\hline & 55688.063 & 3059 & $-74 \pm 62$ & $-64 \pm 68$ & & & \\
\hline \multirow{2}{*}{ HD 93632} & 55687.217 & 2927 & $-152 \pm 50$ & $-120 \pm 62$ & $3318-4593$ & & ND \\
\hline & 55688.198 & 2443 & $-10 \pm 32$ & $5 \pm 68$ & & & \\
\hline \multirow[t]{2}{*}{ CPD-582611 } & 55687.151 & 2631 & $-58 \pm 44$ & $-80 \pm 59$ & $3580-4588$ & & \\
\hline & 55688.125 & 2005 & $-25 \pm 56$ & $-9 \pm 74$ & & & \\
\hline \multirow{4}{*}{ HD 93843} & *55339.099 & 3504 & $-157 \pm 42$ & $-173 \pm 56$ & $3370-5545$ & & PD \\
\hline & 55686.115 & 3230 & $-34 \pm 38$ & $-12 \pm 52$ & & & \\
\hline & 55687.185 & 3385 & $-132 \pm 39$ & $-93 \pm 56$ & & & $\mathrm{CD}$ \\
\hline & 55688.180 & 2647 & $-63 \pm 47$ & $-130 \pm 61$ & & & \\
\hline \multirow[t]{3}{*}{ HD 130298} & *55339.159 & 3037 & $113 \pm 38$ & $193 \pm 62$ & $3573-5284$ & & PD \\
\hline & 55686.347 & 2264 & $-64 \pm 66$ & $-64 \pm 85$ & & & \\
\hline & 55688.261 & 2699 & $59 \pm 55$ & $122 \pm 72$ & & & \\
\hline \multirow[t]{7}{*}{ HD 148937} & *54 550.416 & 2616 & $-276 \pm 88$ & $-145 \pm 104$ & $3210-3599$ & 0.631 & PD \\
\hline & *55 337.285 & 3364 & $-204 \pm 71$ & $-225 \pm 103$ & & 0.529 & \\
\hline & *55339.206 & 2253 & $-290 \pm 85$ & $-389 \pm 129$ & & 0.802 & PD \\
\hline & 55686.370 & 2664 & $-110 \pm 90$ & $-165 \pm 115$ & & 0.172 & $\mathrm{CD}$ \\
\hline & 55687.265 & 3083 & $-87 \pm 74$ & $-137 \pm 95$ & & 0.299 & CD \\
\hline & 55688.285 & 2941 & $-139 \pm 33$ & $-174 \pm 121$ & & 0.444 & CD \\
\hline & 55689.361 & 2522 & $-241 \pm 91$ & $-229 \pm 118$ & & 0.597 & $\mathrm{CD}$ \\
\hline
\end{tabular}

Notes. Entries related to previous measurements are indicated by an asterisk in the second column of the Table. The wavelength region used for the magnetic field measurements is indicated in the sixth column. The seventh column provides the rotation phases for the Of?p star HD 148937, which has a known rotation period of about seven days (Nazé et al. 2010). In the last column ND stands for new detections, PD for previous detections (Hubrig et al. 2008, 2011b), and CD for confirmed detections. All quoted errors are $1 \sigma$ uncertainties.

carried out on single epochs for four out of five stars, only HD 130298 was observed on two different nights. Since the rotation periods for them are unknown, the non-detection of their magnetic fields can probably be explained by the strong dependence of the longitudinal magnetic field on the rotational aspect.

The strongest magnetic fields are detected in the two Of?p stars CPD-28 2561 and HD 148937. Walborn (1973) introduced the Of?p category for massive $\mathrm{O}$ stars displaying recurrent spectral variations in certain spectral lines, sharp emission or P Cygni profiles in He I and the Balmer lines, and strong $\mathrm{C}$ III emission lines around $4650 \AA$. Only five Galactic Of?p stars are presently known: HD 108, NGC 1624-2, CPD-28 2561, HD 148937, and HD 191612 (Walborn et al. 2010), and all of them show evidence for the presence of magnetic fields (Martin et al. 2010; Wade et al. 2012a; Hubrig et al. 2008, 2011b; Donati et al. 2006). The record holder is the faintest star in the sample of Of?p stars, NGC 1624-2, for which a longitudinal magnetic field of the order of $5 \mathrm{kG}$ was recently detected by Wade et al. (2012a).

Besides the small group of Of?p stars, our measurements indicate that the presence of magnetic fields can be expected in stars of different classification categories and at different evolutionary stages, from young main-sequence stars up to the supergiant stages. The different spectral appearance of stars with confirmed and new detections is illustrated in Fig. 1 where we present normalised FORS $1 / 2$ spectra in integral light. Among the whole sample, the two Of?p stars CPD-282561 and HD 148937 exhibit the most conspicuous spectral variability clearly visible in Fig. 1, showing the spectra of these stars obtained at two different epochs. To calculate the rotation phase of the observed spectra of HD 148937, we used the rotation period of $7.03 \mathrm{~d}$ determined by Nazé et al. (2008). The stars with confirmed and new magnetic field detections are discussed in more detail in Sect. 3.

\subsection{SOFIN and HARPS observations}

Four O-type stars, HD 36879, 15 Mon, 9 Sgr, and HD 191612 , previously observed with FORS 1 and SOFIN (Hubrig et al. 2008, 2009a, 2010), were followed-up by nine additional SOFIN 
Table 2. continued.

\begin{tabular}{|c|c|c|c|c|c|c|c|}
\hline Name & MJD & $S / N$ & $\begin{array}{c}\left\langle B_{z}\right\rangle_{\text {all }} \\
{[\mathrm{G}]}\end{array}$ & $\begin{array}{c}\left\langle B_{z}\right\rangle_{\text {hydr }} \\
{[\mathrm{G}]}\end{array}$ & $\begin{array}{l}\text { Spectral } \\
\text { Regions }\end{array}$ & Phase & Comment \\
\hline \multirow[t]{10}{*}{$\zeta \mathrm{Oph}$} & $* 54609.343$ & 4083 & $105 \pm 45$ & $54 \pm 54$ & $3645-4960$ & & $\mathrm{PD}$ \\
\hline & 55686.321 & 3719 & $-164 \pm 62$ & $-218 \pm 70$ & & & $\mathrm{CD}$ \\
\hline & 55687.116 & 3239 & $-83 \pm 63$ & $-90 \pm 81$ & & & \\
\hline & 55687.287 & 2659 & $-153 \pm 75$ & $-222 \pm 97$ & & & \\
\hline & 55687.385 & 2592 & $-101 \pm 88$ & $-84 \pm 104$ & & & \\
\hline & 55688.103 & 1964 & $18 \pm 113$ & $18 \pm 144$ & & & \\
\hline & 55688.240 & 2792 & $95 \pm 68$ & $97 \pm 80$ & & & \\
\hline & 55688.335 & 2226 & $-72 \pm 94$ & $-37 \pm 108$ & & & \\
\hline & 55688.433 & 2486 & $-16 \pm 89$ & $14 \pm 110$ & & & \\
\hline & 55689.349 & 2193 & $58 \pm 90$ & $52 \pm 117$ & & & \\
\hline \multirow{5}{*}{ HD 328856} & *55336.370 & 3239 & $-173 \pm 53$ & $-155 \pm 65$ & $3589-5669$ & & PD \\
\hline & *55 339.223 & 2975 & $-149 \pm 48$ & $-75 \pm 72$ & & & PD \\
\hline & 55686.417 & 2309 & $-43 \pm 48$ & $-96 \pm 70$ & & & \\
\hline & 55687.351 & 2612 & $9 \pm 46$ & $21 \pm 64$ & & & \\
\hline & 55688.301 & 2279 & $-128 \pm 50$ & $-250 \pm 77$ & & & CD \\
\hline \multirow[t]{2}{*}{ CPD-468221 } & 55686.393 & 2389 & $-132 \pm 42$ & $-65 \pm 63$ & $3569-5158$ & & ND \\
\hline & 55688.319 & 2195 & $-83 \pm 45$ & $-69 \pm 68$ & & & \\
\hline \multirow[t]{2}{*}{ HD 150958} & 55686.431 & 1957 & $133 \pm 65$ & $122 \pm 118$ & $3695-4974$ & & \\
\hline & 55687.372 & 2206 & $-105 \pm 63$ & $-74 \pm 106$ & & & \\
\hline \multirow{3}{*}{ HD 153426} & *55336.338 & 3446 & $-27 \pm 53$ & $-10 \pm 62$ & $3650-4874$ & & \\
\hline & *55339.246 & 3136 & $-171 \pm 55$ & $-275 \pm 70$ & & & PD \\
\hline & 55687.434 & 2318 & $19 \pm 65$ & $62 \pm 82$ & & & \\
\hline \multirow[t]{2}{*}{ HD 153919} & *55337.341 & 2727 & $-213 \pm 68$ & $-119 \pm 95$ & $3680-4452$ & & PD \\
\hline & 55687.421 & 2727 & $26 \pm 82$ & $152 \pm 102$ & & & \\
\hline \multirow{2}{*}{ HD 154643} & *55339.340 & 3382 & $110 \pm 34$ & $121 \pm 52$ & $3376-4910$ & & PD \\
\hline & 55689.374 & 2925 & $73 \pm 42$ & $163 \pm 58$ & & & \\
\hline HD 156041 & 55689.393 & 1501 & $71 \pm 69$ & $138 \pm 109$ & $3689-5663$ & & \\
\hline \multirow[t]{2}{*}{ HD 156154} & *55337.358 & 2753 & $-118 \pm 38$ & $-167 \pm 54$ & $3617-4974$ & & PD \\
\hline & 55688.418 & 2498 & $19 \pm 54$ & $117 \pm 72$ & & & \\
\hline \multirow[t]{2}{*}{ HD 157857} & 55687.401 & 3035 & $-110 \pm 46$ & $-226 \pm 61$ & $3383-4974$ & & ND \\
\hline & 55688.348 & 2785 & $-116 \pm 50$ & $-51 \pm 72$ & & & \\
\hline HD 170580 & 55688.403 & 2579 & $-55 \pm 31$ & $-77 \pm 47$ & $3343-6196$ & & \\
\hline HD 170783 & 55688.389 & 3016 & $-19 \pm 34$ & $-2 \pm 43$ & $3326-5885$ & & \\
\hline HD 172175 & 55688.370 & 2210 & $-73 \pm 94$ & $-79 \pm 124$ & $3670-4480$ & & \\
\hline
\end{tabular}

spectropolarimetric observations from 2008 September and 2010 July. Spectral classifications of these stars are presented in Table 1. Among these stars, only for the Of?p star HD 191612 was the presence of a magnetic field of the order of a few hundred Gauss previously reported by Donati et al. (2006). We used the low-resolution camera $(R \approx 30000)$ of the echelle spectrograph SOFIN (Tuominen et al. 1999) mounted at the Cassegrain focus of the NOT. With the $2 \mathrm{~K}$ Loral CCD detector we registered 40 echelle orders partially covering the range from 3500 to $10000 \AA$ with a length of the spectral orders of about $140 \AA$ at $5500 \AA$. The polarimeter is located in front of the entrance slit of the spectrograph and consists of a fixed calcite beam splitter aligned along the slit and a rotating super-achromatic quarterwave plate. Two spectra polarised in opposite sense are recorded simultaneously for each echelle order providing sufficient separation by the cross-dispersion prism. Two to four sub-exposures with the quarter-wave plate angles separated by $90^{\circ}$ were used to derive circularly polarised spectra. A detailed description of the SOFIN spectropolarimeter and its polarimetric data reduction is given in Ilyin (2012).

The spectra were reduced with the $4 \mathrm{~A}$ software package (Ilyin 2000). Bias subtraction, master flat-field correction, scattered light subtraction, and weighted extraction of spectral orders comprise the standard steps of spectrum processing. A ThAr spectral lamp is used for wavelength calibration, taken before and after each target exposure to minimise temporal variations in the spectrograph. The typical $\mathrm{S} / \mathrm{N}$ for observations of $9 \mathrm{Sgr}$ is between 280 and 380, while for HD 36879 and HD 191612 it is about 200. The highest $\mathrm{S} / \mathrm{N}$ of 440 was achieved for $15 \mathrm{Mon}$.

In addition, the two O-type stars, HD 151804 and 9 Sgr, previously analysed using FORS 1 data (Hubrig et al. 2008) were observed on 2011 May 25 and 26 with a S/N of about 280 with the HARPSpol polarimeter (Snik et al. 2011) feeding the HARPS spectrometer (Mayor et al. 2003) at the ESO $3.6 \mathrm{~m}$ telescope on La Silla within the framework of the programme 187.D-0917(A). This HARPS spectropolarimetric material became recently publically available in the ESO archive. The HARPS archive spectra cover the wavelength range 3780-6913 , with a small gap around $5300 \AA$. Each HARPSpol observation is usually split into four to eight sub-exposures, obtained with four different orientations of the quarter-wave retarder plate relative to the beam splitter of the circular polarimeter. The reduction was performed using the HARPS data reduction software available at the ESO headquarters in Germany. The Stokes $I$ and $V$ parameters were derived following the ratio method described by Donati et al. (1997), ensuring in particular that all spurious signatures are removed at first order. Null polarisation spectra (labelled with $n$ in Table 3 ) were calculated by combining the sub-exposures in such a way that the polarisation cancels out, allowing us to verify that no spurious signals are present in the data.

HARPS and SOFIN spectra have been measured using the moment technique previously introduced by Mathys who discussed at length the diagnostic potential of high-resolution 
S. Hubrig et al.: Exploring the origin of magnetic fields in massive stars. II.

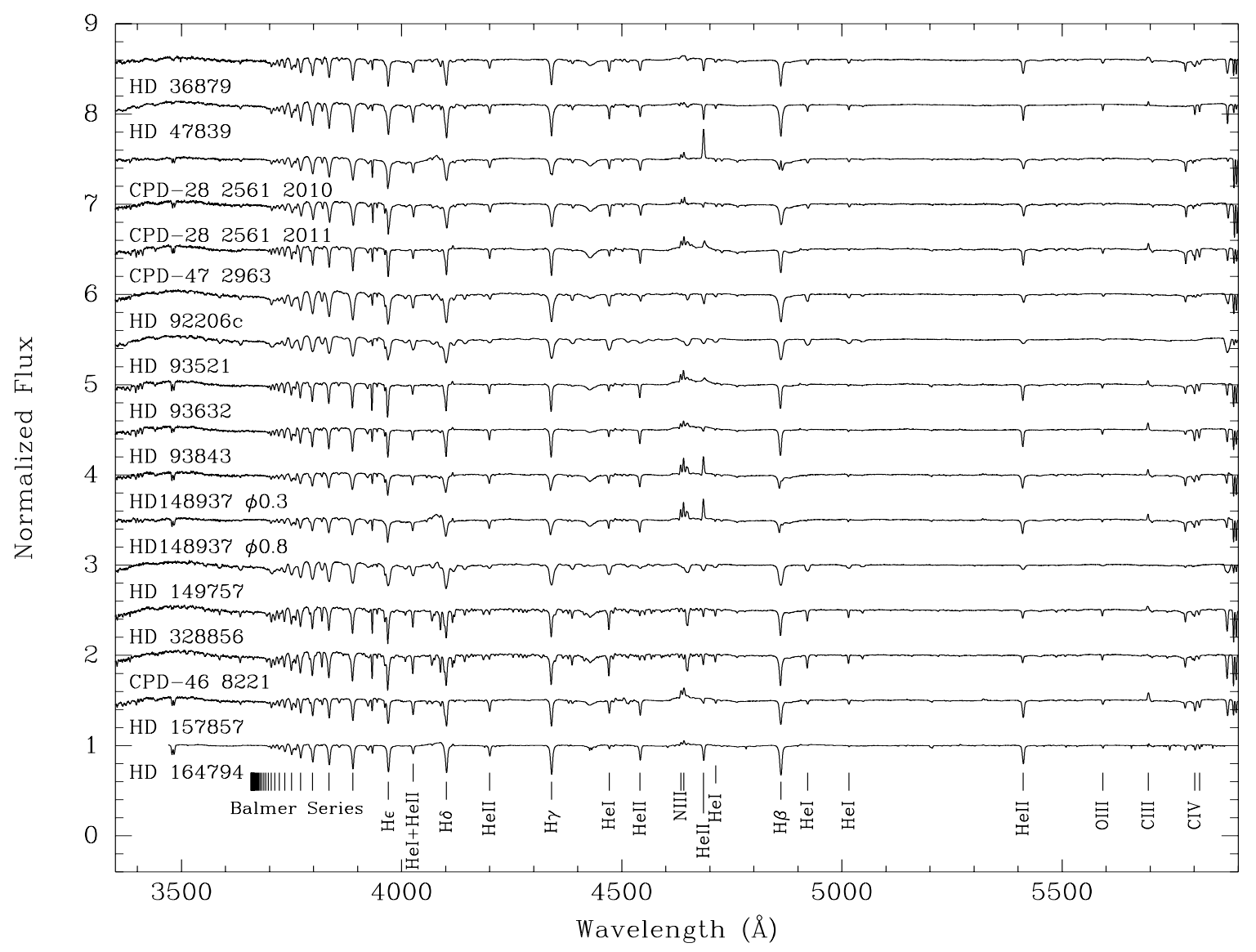

Fig. 1. Normalised FORS 1/2 Stokes $I$ spectra of O-type stars with detected magnetic fields observed from 2005 to 2011 . Well known spectral lines are indicated; all Balmer lines from the Balmer jump to $\mathrm{H} \beta$ are visible. The spectra were offset from 1 by multiples of 0.5 for clarity.

circularly polarised spectra using this technique in numerous papers (e.g., Mathys 1993, 1995a,b). Wavelength shifts between right- and left-hand side circularly polarised spectra are interpreted in terms of a longitudinal magnetic field $\left\langle B_{z}\right\rangle .\left\langle B_{z}\right\rangle$ is obtained by performing a least-squares fit, forced through the origin, of the wavelength shifts $\lambda_{\mathrm{R}}-\lambda_{\mathrm{L}}$, as a function of $2 \bar{g} \Delta \lambda_{\mathrm{z}}$. In this process, the individual measurements are weighted by the respective $1 / \sigma^{2}\left(\lambda_{R}-\lambda_{L}\right)$. The uncertainty of the derived value of the longitudinal magnetic field is estimated by its standard error $\sigma\left(\left\langle B_{z}\right\rangle\right)$, derived from the least-squares analysis (e.g., Mathys $\&$ Hubrig 1995). The results of the longitudinal magnetic field measurements using mostly He I and He II lines are presented in Table 3.

The high-resolution spectropolarimetric observations confirm our previous detections of magnetic fields in the stars HD 36879 and 9 Sgr. The magnetic nature of the slowly rotating Of?p star HD $191612(P=538 \mathrm{~d})$ was for the first time reported by Donati et al. (2006) who observed it over four nights in 2005 June using the ESPaDOnS spectropolarimeter installed on the 3.6-m Canada-France-Hawaii Telescope and announced the detection of a mean longitudinal field $\left\langle B_{z}\right\rangle=$ $-220 \pm 38 \mathrm{G}$. Hubrig et al. (2010) reported on a single measurement of the magnetic field of HD 191612, $\left\langle B_{z}\right\rangle=450 \pm 153 \mathrm{G}$, using SOFIN observations acquired in 2008 September at rotational phase 0.43 . Wade et al. (2011) presented 13 new field measurements of this star demonstrating that the magnetic data can be modelled as a periodic, sinusoidal signal with a period of $538 \mathrm{~d}$ inferred from spectroscopy. The authors mention in their work that the SOFIN observation of the longitudinal field of HD 191612, $\left\langle B_{z}\right\rangle=450 \pm 153 \mathrm{G}$, does not agree very well with the observed field variation, which corresponds to (an essentially) consistently negative longitudinal magnetic field. To check the consistency of our measurements we re-analysed the previous SOFIN observations using an extended list of blend free spectral lines with known atomic parameters. The obtained results, $\left\langle B_{z}\right\rangle=357 \pm 58 \mathrm{G}$, indicates, however, that contrary to the results of Wade et al. (2011), the magnetic field of HD 191612 is indeed rather strongly positive in the phase 0.43 . Clearly, more magnetic field observations of this star are needed to properly characterise the variation of the longitudinal magnetic field and the magnetic field geometry. In Fig. 2 we present our SOFIN observations together with those published by Wade et al. (2011).

The supergiant HD 151804 was previously observed three times in 2005 with FORS 1 (Hubrig et al. 2008). No field detection at a significance level of $3 \sigma$ was achieved indicating that the field should be rather weak if present at all. Our measurement $\left\langle B_{z}\right\rangle=-104 \pm 38 \mathrm{G}$ based on HARPSpol observations confirms these previous results.

\section{Results for individual targets with detected magnetic fields}

\subsection{HD 36879}

A magnetic field at a $3.5 \sigma$ significance level in HD 36879 was for the first time detected in 2007 using FORS 1 observations (Hubrig et al. 2008). Our most recent high-resolution SOFIN observations in 2009 December confirm the previous detection and 
Table 3. Magnetic field measurements of five O-type stars using SOFIN and HARPS observations.

\begin{tabular}{|c|c|c|c|c|}
\hline MJD/HJD & Phase & $\left\langle B_{z}\right\rangle_{\text {all }}[\mathrm{G}]$ & $\left\langle B_{z}\right\rangle_{\mathrm{n}}[\mathrm{G}]$ & Instr. \\
\hline \multicolumn{5}{|c|}{ HD 36879} \\
\hline $\begin{array}{l}\text { *54345.389 } \\
2454723.737 \\
2455195.615\end{array}$ & & $\begin{array}{r}180 \pm 52 \\
-125 \pm 78 \\
-276 \pm 89 \\
\end{array}$ & $\begin{array}{r}53 \pm 75 \\
-64 \pm 92 \\
\end{array}$ & $\begin{array}{l}\text { FORS } 2 \\
\text { SOFIN } \\
\text { SOFIN } \\
\end{array}$ \\
\hline \multicolumn{5}{|c|}{15 Mon } \\
\hline $\begin{array}{l}* 54609.968 \\
2455201.553 \\
\end{array}$ & & $\begin{array}{r}134 \pm 52 \\
-162 \pm 37 \\
\end{array}$ & $-62 \pm 42$ & $\begin{array}{l}\text { FORS } 2 \\
\text { SOFIN }\end{array}$ \\
\hline \multicolumn{5}{|c|}{ HD 151804} \\
\hline $\begin{array}{l}\text { *53 476.369 } \\
\text { *53 571.025 } \\
\text { *53596.096 } \\
55708.356 \\
\end{array}$ & & $\begin{array}{r}-151 \pm 90 \\
68 \pm 65 \\
82 \pm 46 \\
-104 \pm 38 \\
\end{array}$ & $-31 \pm 36$ & $\begin{array}{l}\text { FORS } 2 \\
\text { FORS } 2 \\
\text { FORS } 2 \\
\text { HARPS } \\
\end{array}$ \\
\hline \multicolumn{5}{|c|}{$9 \mathrm{Sgr}$} \\
\hline $\begin{array}{l}\text { *53 520.357 } \\
\text { *53 594.119 } \\
\text { *53 595.096 } \\
2455074.387 \\
2455075.384 \\
2455078.396 \\
2455081.382 \\
55707.323 \\
\end{array}$ & & $\begin{array}{r}-114 \pm 66 \\
211 \pm 57 \\
-165 \pm 75 \\
187 \pm 56 \\
242 \pm 74 \\
238 \pm 59 \\
-265 \pm 65 \\
210 \pm 42 \\
\end{array}$ & $\begin{array}{r}-33 \pm 60 \\
72 \pm 76 \\
66 \pm 66 \\
-42 \pm 62 \\
39 \pm 41 \\
\end{array}$ & $\begin{array}{l}\text { FORS } 2 \\
\text { FORS } 2 \\
\text { FORS } 2 \\
\text { SOFIN } \\
\text { SOFIN } \\
\text { SOFIN } \\
\text { SOFIN } \\
\text { HARPS }\end{array}$ \\
\hline \multicolumn{5}{|c|}{ HD 191612} \\
\hline $\begin{array}{l}* 2454721.347 \\
2455395.592 \\
2455407.583\end{array}$ & $\begin{array}{l}0.43 \\
0.68 \\
0.68\end{array}$ & $\begin{array}{r}357 \pm 58 \\
-144 \pm 34 \\
-154 \pm 63\end{array}$ & $\begin{array}{r}67 \pm 65 \\
-39 \pm 36 \\
-67 \pm 70\end{array}$ & $\begin{array}{l}\text { SOFIN } \\
\text { SOFIN } \\
\text { SOFIN }\end{array}$ \\
\hline
\end{tabular}

Notes. Already published measurements are marked by asterisks. All quoted errors are $1 \sigma$ uncertainties.

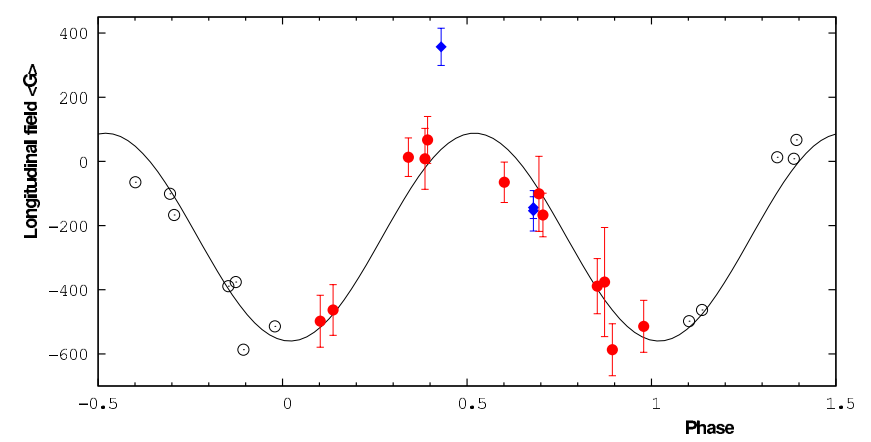

Fig. 2. Longitudinal magnetic field variation of the Of?p star HD 191612 according to the $537.6 \mathrm{~d}$ period determined by Howarth et al. (2007). Red symbols correspond to ESPaDOnS observations, while blue symbols are our SOFIN measurements (Hubrig et al. 2010, and Table 3). Note that the measurement errors for both ESPaDOnS and SOFIN observations are of similar order.

reveal a change of the field polarity. Walborn (2006) reports that this star shows unexplained spectral peculiarities and/or variations, among them peculiar narrow, variable Si IV emission lines at $\lambda 1394$ and $\lambda 1403$ (e.g. Walborn \& Panek 1984). Distinct spectral variability is also discovered in Fe III and the blended lines $\mathrm{C}$ III/O II using our two high-resolution SOFIN spectra obtained on two different epochs. The variations of Fe III and $\mathrm{C} \mathrm{III/O} \mathrm{II} \mathrm{line} \mathrm{profiles} \mathrm{are} \mathrm{presented} \mathrm{in} \mathrm{Fig.} \mathrm{3.} \mathrm{Interestingly,}$ He I lines appear slightly split. This appearance presented in Fig. 4 could possibly indicate the presence of He spots on the stellar surface.
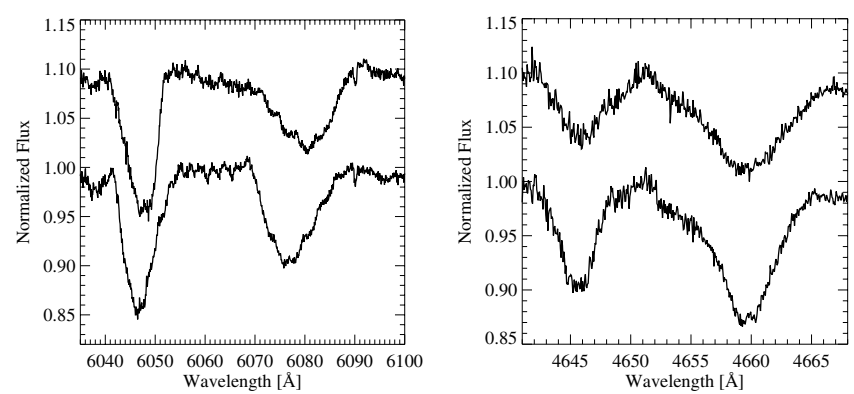

Fig. 3. Variable line profiles in the SOFIN spectra of HD 36879 obtained at two different epochs. Left panel: spectral variability of the Fe III line profile at $\lambda 6048$ and the blend Fe III+He II at $\lambda 6078$. Right panel: spectral variability of line profiles presenting blends of the $\mathrm{C}$ III and $\mathrm{O}$ II lines at $\lambda 4647$ and $\lambda 4660$.
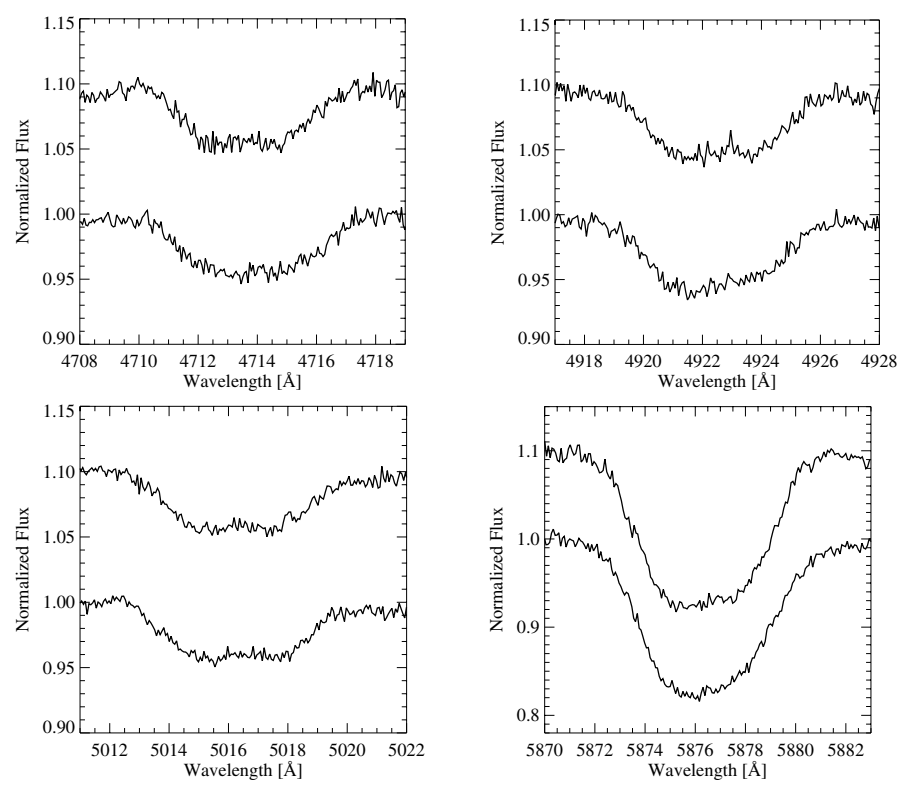

Fig. 4. Line profiles of $\mathrm{He} \mathrm{I}$ in the spectra of HD 36879 obtained at two different epochs. From top left to bottom right we show the lines He I $\lambda 4713, \lambda 4922, \lambda 5015$, and $\lambda 5876$.

\subsection{Mon}

Hubrig et al. (2011b) showed that this star is a probable member of the young, rich open cluster NGC 2264 located in the Monoceros OB 1 association. From the spatial distribution of $\mathrm{H} \alpha$ emission stars, Sung et al. (2008) have concluded that the young open cluster NGC 2264 can be subdivided into three star forming regions (SFRs): two active SFRs - the region around the bright emission nebula in the southwest of the brightest star $\mathrm{S}$ Mon and the region to the north of the famous cone nebula - and surrounding these an elliptical-shaped halo region. The study of Teixeira et al. (2012) indicates that NGC 2264 has undergone more than one star-forming event, where the unembedded, insubstantial inner disk population and embedded thick inner disk population appear to have formed in separate episodes. Markova et al. (2004) consider 15 Mon as a Galactic O-type star with a mass of $32 M_{\odot}$ and $T_{\text {eff }}=37500 \mathrm{~K}$. Speckle measurements of 15 Mon indicated the presence of a companion of spectral type O9.5 V n (e.g. Gies et al. 1993). In very high S/N spectra becomes the companion noticeable by very broad underlying components to the strong He I lines. According to Kaper et al. (1996) and Walborn (2006) shows this star distinct peculiarities in the spectra, which could be typical for stars possessing 

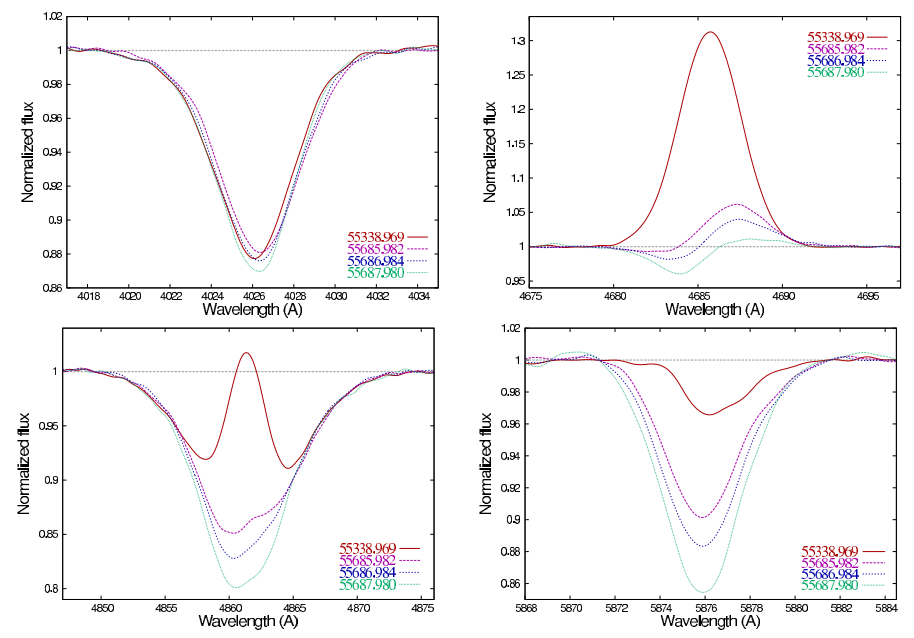

Fig. 5. Distinct variability observed in the spectra of CPD-282561 in the He I $\lambda 4026$ (top left), He II $\lambda 4686$ (top right), $\mathrm{H} \beta$ (bottom left), and He I $\lambda 5876$ (bottom right) lines.

magnetic fields. The X-ray luminosity based on Chandra observations is $\log \left(L_{\mathrm{X}}\right)=31.82$ and the ratio of $\mathrm{X}$-ray to bolometric luminosity $\log \left(L_{\mathrm{X}} / L_{\mathrm{bol}}\right)=-6.63$ is slightly above the average (Ramirez et al. 2004; Nazé 2009), as may be expected from a magnetic star. The high-resolution X-ray spectrum of 15 Mon is typical for a star with its spectral type and, apparently, not especially hard (Walborn et al. 2009).

The previously measured magnetic field using the full FORS 1 spectrum coverage was of positive polarity at a $2.6 \sigma$ significance level (Hubrig et al. 2009a). A weak magnetic field was detected in this star at a significance level of $4.4 \sigma$ using SOFIN observations. No contribution of the secondary component was detected in our FORS 1 and SOFIN spectra (see also Fig. 1).

\section{3. $C P D-282561$}

Our previous observations of the Of?p star CPD-282561 enabled us to detect a magnetic field at the $3.1 \sigma$ level using the whole spectrum and at the $3.2 \sigma$ level using Balmer lines (Hubrig et al. 2011b). Our new spectropolarimetric observations of this star, recently reported by us in a short IBVS article (Hubrig et al. 2012a) indicate that the magnetic field is variable. Due to the relative faintness of CPD-282561 with $V=10.1$, it was only scarcely studied in the past. Levato et al. (1988) acquired radial velocities of $35 \mathrm{OB}$ stars with carbon, nitrogen, and oxygen anomalies and found variability of a few emission lines with a probable period of 17 days. Walborn et al. (2010) mentioned that CPD-28 2561 undergoes extreme spectral transformations very similar to those of HD 191612, on a time scale of weeks, inferred from the variable emission intensity of the C III $\lambda \lambda$ 4647-4650-4652 triplet. Our spectropolarimetric observations on three consecutive nights in 2011 reveal strong variations in several hydrogen and helium line profiles. The variation of He I $\lambda 4026$, He I $\lambda 2876$, and of $\mathrm{H} \beta$ on such a short time scale were not reported in the literature before. A few examples of the detected spectral line profile variations are presented in Fig. 5. The contribution of a variable emission component is well visible in three lines. The profile of the He I $\lambda 4026$ line appears only weakly variable. These variations have an amplitude of about $1-2 \%$ of the continuum level and can likely be explained by rotation modulation. The line He II $\lambda 4686$ exhibited strong emission during our observations in 2010, which transforms to a much weaker PCygni line profile with a weak blue shifted absorption in 2011. A contribution of the emission component is clearly seen in the profile of the He I $\lambda 5876$ line in 2010 , but its presence can only be suspected in the variable line depth observed in 2011. A strong emission component is well visible in the line centre of $\mathrm{H} \beta$ in 2010 . The shape of the line profile of $\mathrm{H} \beta$ is similar to the behaviour of the $\mathrm{H} \alpha$ line profile in the spectra of 19 Cep presented in Fig. 13 of Kaper et al. (1997) and in Fig. 1 of Kholtygin et al. (2003).

CPD-28 2561 is an intrinsically bright X-ray source as detected by the Rosat X-ray observatory. Its ratio of X-ray to bolometric luminosity $\left(\log L_{\mathrm{X}} / L_{\mathrm{bol}} \approx-6\right)$ makes it unusually X-ray bright compared to other O-type stars (Chlebowski 1989; Oskinova 2005). The hardness ratio $(H R 1 \approx 0.04 \pm 0.4)$ indicates that its X-ray emission can be harder than is typical in single O-type stars. This provides indirect support for the presence of a magnetic field on the star, strong enough to confine the stellar wind (Babel \& Montmerle 1997).

\section{4. $C P D-472963$}

For the star CPD-47 2963, we confirm our previous 3.1 $\sigma$ detection by measuring all absorption lines in the FORS 2 spectra. The magnetic field of this star is variable and shows a change of polarity over our four-night observing run. According to Walborn et al. (2010), CPD-47 2963 belongs to the Ofc category, which consists of normal spectra with C III $\lambda \lambda$ 4647-4650-4652 emission lines of comparable intensity to those of the Of defining lines N III $\lambda \lambda$ 4634-4640-4642. The origin of the magnetic field in this star probably differs from those of other magnetic O-type stars, because non-thermal radio emission, which is frequently observed in binary systems with colliding winds, was detected by Benaglia et al. (2001). On the other hand, the membership of CPD-47 2963 to a binary or multiple system has not yet been investigated.

\section{5. $H D 92206 c$}

The presence of a variable magnetic field up to a $4.4 \sigma$ significance level in HD 92206c is documented here for the first time. This star is a probable member of the open cluster NGC 3324 located inside of a partial ring of nebulosity northwest of the Carina Nebula. The assessment of the membership using astrometric catalogues with the highest quality kinematic and photometric data currently available is described in Sect. 4. According to the Galactic O Star Catalogue (Maíz-Apellániz et al. 2004) belongs HD 92206c to the triple system HD 92206abc. An analysis of the distribution of the ionised and neutral material associated with the H II region Gum 31 by Cappa et al. (2008) suggested that the excitation sources of this region are the brightest stars in the open cluster NGC 3324 with the triple system HD 92206abc considered as the brightest cluster member. The detection of protostellar candidates almost coincident in position with the open cluster NGC 3324 indicates that stellar formation is ongoing in this region.

Walborn (1982) reports that the spectrum of HD 92206c displays very strong, broad hydrogen lines, possibly similar to those in the Orion Trapezium cluster, in particular $\theta^{1}$ Ori $C$, and the appearance of these lines is indicative of extreme youth. A search for a magnetic field was carried out in another member of this cluster, the bright A0-type supergiant HD 92207. According to Hubrig et al. (2012b), this star also possesses a magnetic field. 


\section{6. $H D 93521$}

A longitudinal magnetic field of the order of $200-300 \mathrm{G}$ is detected in this star at a significance level of $3.2 \sigma$. This high Galactic latitude 09.5 Vp star with $v \sin i=390 \mathrm{~km} \mathrm{~s}^{-1}$ (Rauw et al. 2008) is one of the fastest rotators in our sample, along with $\zeta$ Oph. It is located at an unusually high Galactic latitude of 62.15 and displays prominent line profile variability in the optical and UV domain (Rauw et al. 2008). In the most recent work by Rauw et al. (2012a), the authors report that $\mathrm{He}$ and $\mathrm{N}$ are found to be overabundant and suggest that despite some ambiguities on the runaway status of the star it is likely that the star is a Population I massive O-type star that was ejected from the Galactic plane either through dynamical interactions or as the result of a supernova event in a binary system.

HD 93521 is one of the few O-type stars known to exhibit stellar pulsations. In this star, two frequencies of about $2-3 \mathrm{~h}$ are detected and interpreted as due to high-degree non-radial pulsation modes (Howarth \& Reid 1993; Rauw et al. 2008).

The X-ray observations of this star have been summarised by Rauw et al. (2012a). They concluded that the stellar X-ray spectrum is consistent with a normal late O-type star although with subsolar metallicity. No trace of a compact companion was found in the X-ray data. The ratio of X-ray to bolometric luminosity is $\log \left(L_{\mathrm{X}} / L_{\mathrm{bol}}\right)=-7.1 \ldots-7.0$ and the stellar X-ray luminosity $L_{X}=5 \times 10^{29} \mathrm{erg} \mathrm{s}^{-1}$ is rather low. Such low X-ray luminosities are also observed in other magnetic stars. Oskinova et al. (2011) showed, that relatively low X-ray luminosities can be explained in the framework of the magnetically confined wind shock model if the stellar wind strength is correctly derived from an optical and UV spectroscopic analysis. Significantly, Rauw et al. (2012a) show that besides a softer plasma component with $k T_{1}=0.3 \mathrm{keV}$, which is typical for normal O-stars, a harder component with $k T_{2}=3 \mathrm{keV}$ is present in the X-ray spectrum of HD 93521. Recently, Ignace et al. (2013) showed that such a hard component may serve as an indicator for the presence of a stellar magnetic field.

\subsection{HD 93632 and HD 93843}

A weak negative magnetic field in HD 93632, just at a significance level of $3 \sigma$ was detected in one out of two FORS 2 observations. Walborn (1973) reported that both HD 93632 and HD 93843 appear slightly variable in their spectral types. Interestingly, also HD 93843, similar to HD 93632, exhibits a weak magnetic field. It's presence is confirmed in our new observations. According to Walborn et al. (2010), the star HD 93843 belongs to the Ofc category. Prinja et al. (1998) monitored the stellar wind of this star using IUE time series. They identified systematic changes in the absorption troughs of the Si IV and $\mathrm{NV}$ resonance lines with a repeatability of wind structures with a period of 7.1 days. The authors suggested at that time that a magnetic field might help to explain the cyclical wind perturbation. Both stars have been observed during the Chandra X-ray survey of the Carina Nebula (Townsley et al. 2011). For HD 93632 is the X-ray luminosity $\log L_{X}=31.7$. The ratio of $\mathrm{X}$-ray to bolometric luminosity is small, only $\log \left(L_{\mathrm{X}} / L_{\mathrm{bol}}\right)=-7.7$. A one-temperature fit to the X-ray spectrum shows a slightly higher than average temperature $k T=$ 0.56 (Nazé et al. 2011). For HD 93843, the X-ray luminosity is $\log L_{\mathrm{X}}=32.1$. The ratio of X-ray to bolometric luminosity is standard, $\log \left(L_{\mathrm{X}} / L_{\mathrm{bol}}\right)=-7.1$. A one-temperature fit to the $\mathrm{X}$-ray spectrum derives a spectral temperature $k T=0.3$, which is lower than in HD 93632 (Nazé et al. 2011).

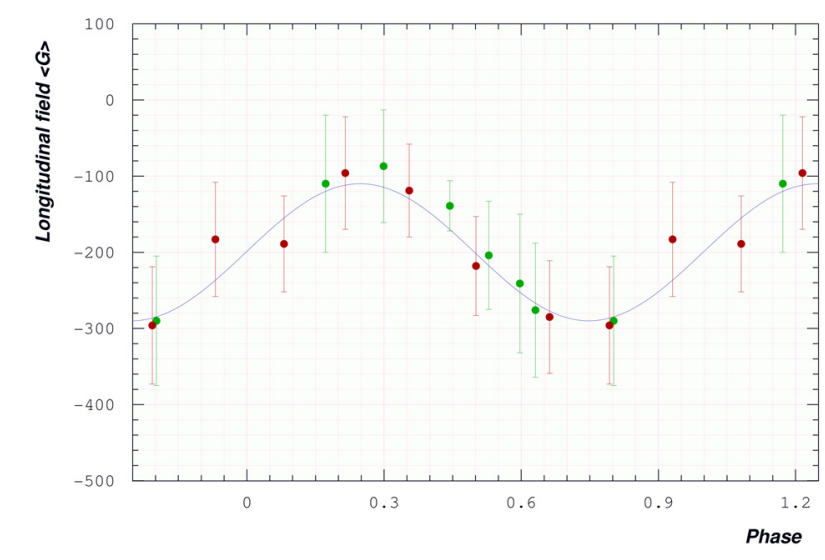

Fig. 6. Longitudinal magnetic field variation of the Of?p star HD 148937 according to the $7.032 \mathrm{~d}$ period determined by Nazé et al. (2010). Red symbols correspond to the ESPaDOnS observations (Wade et al. 2012b), while green symbols are our FORS 1 and FORS 2 measurements (Hubrig et al. 2008, 2011b and present paper). Note that the measurement errors for both ESPaDOnS and FORS $1 / 2$ observations are of similar order. One more measurement (not shown in this figure) was obtained in 2010 on May 22, but ordinary and extraordinary beams in the FORS 2 setup were overlapping in this exposure due to problems with slitlets.

\section{8. $H D 148937$}

The first detection of a mean longitudinal magnetic field $\left\langle B_{z}\right\rangle=$ $-254 \pm 81 \mathrm{G}$ in the Of?p star HD 148937 using FORS 1 at the VLT was reported by Hubrig et al. (2008). An extensive multiwavelength study of HD 148937 was carried out by Nazé et al. (2008), who detected small-scale variations of He II $\lambda 4686$ and the Balmer lines with a period of seven days and an overabundance of nitrogen by a factor of four compared to the Sun. The periodicity of spectral variations in hydrogen and helium lines was re-confirmed using additional higher resolution spectroscopic material indicating the similarity to the other Of?p stars HD 108 and HD 191612 (Nazé et al. 2010). Our new and old observations of HD 148937 are presented in Fig. 6 together with the ESPaDOnS observations obtained at the CFHT by Wade et al. (2012b). This figure demonstrates the excellent potential of FORS 2 for the detection and investigation of magnetic fields in massive stars: While an exposure time of $21.5 \mathrm{~h}$ at the CFHT was necessary to obtain seven binned measurements, the exposure time for FORS 2 observations accounted only for two to four minutes and only $2.3 \mathrm{~h}$ were used for our observations at six different epochs including telescope presets and the usual overheads for readout time and retarder waveplate rotation.

\section{9. $\zeta O p h$}

The star $\zeta$ Ophiuchi is a well-known rapidly rotating runaway star with extremely interesting characteristics. This star exhibits a wonderful bow shock as evident from IR Spitzer IRAC observations and undergoes episodic mass loss seen as emission in $\mathrm{H} \alpha$. It is possible that $\zeta$ Oph rotates with almost break-up velocity with $v \sin i=400 \mathrm{~km} \mathrm{~s}^{-1}$ (Kambe et al. 1993). $\zeta$ Oph is the second target of our sample with detected non-radial pulsations with periods of hours, first discovered from ground-based observations (e.g. Kambe et al. 1997). Later, the star was monitored by the MOST satellite, which revealed at least a dozen significant pulsation frequencies between 1 and $10 \mathrm{~d}^{-1}$, typical of $\beta$ Cep-like pulsation modes (Walker et al. 2005). 
S. Hubrig et al.: Exploring the origin of magnetic fields in massive stars. II.

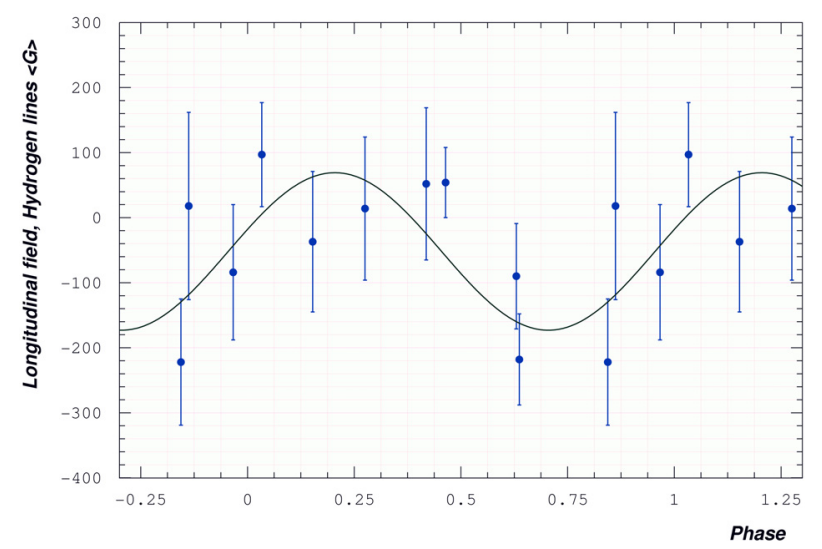

Fig. 7. Phase diagram for the best sinusoidal fit corresponding to the period of 0.8 days for the longitudinal magnetic field measurements using hydrogen lines for $\zeta$ Oph.

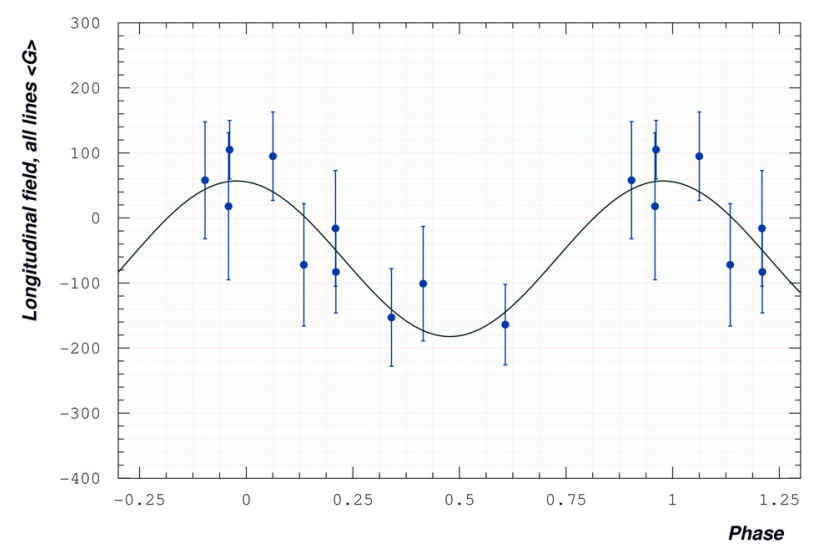

Fig. 8. Phase diagram for the best sinusoidal fit corresponding to the period of 1.3 days for the longitudinal magnetic field measurements using the whole spectrum for $\zeta \mathrm{Oph}$.

The first detection of a mean longitudinal magnetic field in this star was announced by Hubrig et al. (2011c). Nine additional FORS 2 spectropolarimetric observations showing a change of polarity have been obtained over all four observing nights. The rotation period of this star is still uncertain: Oskinova et al. (2001) studied ASCA observations that covered just the expected rotation period of the star of about $0.8-1.0 \mathrm{~d}$. A X-ray flux variability with $\sim 20 \%$ amplitude in the ASCA passband $(0.5-10 \mathrm{keV})$ with a period of $0.77 \mathrm{~d}$ was reported. The X-ray spectra of $\zeta$ Oph are not especially hard as found from Chandra HETGS and Suzaku data (Waldron \& Cassinelli 2007; Walborn et al. 2009). The variation period is also not well-defined in our observations. The resulting periodograms for the magnetic field measurements using hydrogen lines shows a dominating peak corresponding to a period of about $0.8 \mathrm{~d}$, while the measurements using the whole spectrum indicate a rotation period of about $1.3 \mathrm{~d}$. In Fig. 7 we present the magnetic field variation using hydrogen lines phased with the period of 0.8 days, while Fig. 8 refers to magnetic field variations determined from all lines phased with 1.3 days. Interestingly, just a few months ago Pollmann (2012) presented equivalent width changes of He I $\lambda 6678$ with a period of $0.643 \mathrm{~d}$, which is roughly half of the magnetic period of $1.3 \mathrm{~d}$ determined using the whole spectral region. For the rotation period of $\sim 1.3 \mathrm{~d}$ the equivalent width of the He I line would display a double wave. Such a behaviour of He I lines is frequently found in He variable early-type Bp stars (e.g. Briquet et al. 2004). Clearly, more

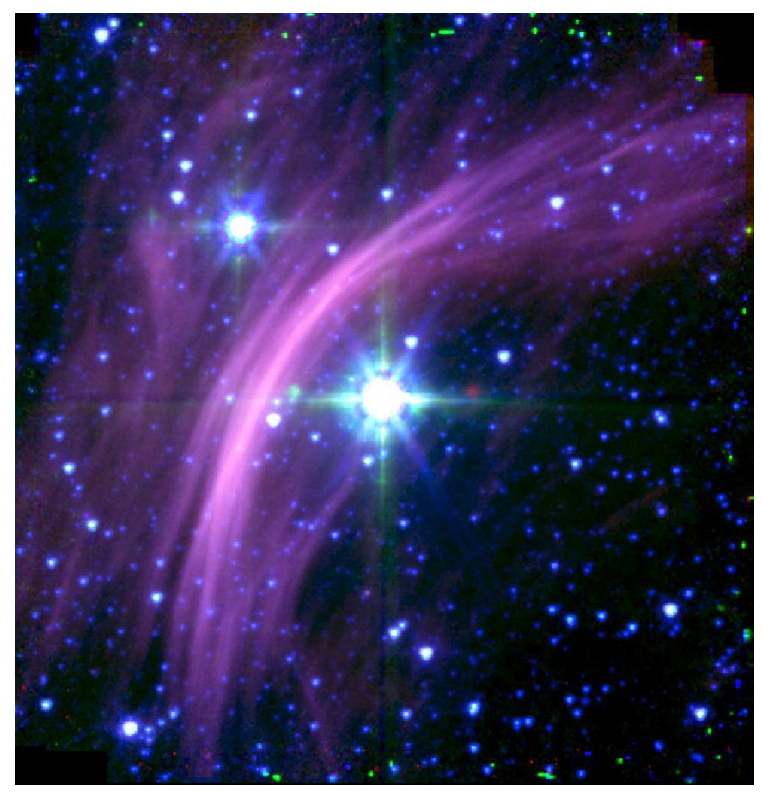

Fig. 9. Combined IR Spitzer IRAC (3.6 $\mu \mathrm{m}$ blue, $4.5 \mu \mathrm{m}$ green, $8.0 \mu \mathrm{m}$ red) image of the bow shock around Vela X-1. Archival data have been used (AOR 17858304, PI: R. Iping). Image size is $5^{\prime} \times 5^{\prime}$.

measurements are needed to determine the true periodicity of the magnetic field in this star.

A bow-shock nebula is also present close to the high mass $\mathrm{X}$-ray binary Vela X-1, consisting of a pulsar in an eccentric ( $e=0.09, P_{\text {orb }}=8.96 \mathrm{~d}$ ) orbit around the here studied B0.5 supergiant star HD77581. In Fig. 9 we present an image of the bow-shock nebula based on archival Spitzer IRAC maps (AOR 17858304, PI R. Iping). The mass-loss rate $(\dot{M} \approx$ $10^{-6} M_{\odot} \mathrm{yr}^{-1}$ ) for Vela X-1 is well constrained from its accretion powered X-ray luminosity (e.g., Fürst et al. 2010; Oskinova et al. 2012). Contrary to our study of $\zeta$ Oph, no magnetic field detection at a significance level of $3 \sigma$ has been achieved in Vela X-1 during our observing run. However, we should keep in mind that the spectral behaviour of HD 77581 is much more complex than that of $\zeta$ Oph due to the presence of bumps and wiggles in the line profiles, and an impact of tidal effects producing orbital phase-dependent variations in the line profiles leading to asymmetries such as extended blue or red wings (Koenigsberger et al. 2012). Furthermore, Kreykenbohm et al. (2008) detected flaring activity and temporary quasi-periodic oscillations in INTEGRAL X-ray observations.

\subsection{HD 328856}

Our new observations confirm the presence of a magnetic field in this star. As we already showed in our previous work on the basis of photometric membership probability, HD 328856 is a member of the compact open cluster Hogg 22 in the Ara region at an age of $5 \mathrm{Myr}$ and a distance of about $1300 \mathrm{pc}$ (Hubrig et al. 2011b). On the other hand, its proper motions indicate that HD 328856 is not fully co-moving with the other cluster members, deviating from the cluster mean proper motion by $\sim 2 \sigma$ (for more details of the membership probabilities, see Kharchenko et al. 2004).

\subsection{1. $C P D-468221$}

A search for the presence of a magnetic field in this star was carried out for the first time. A weak mean longitudinal field was 
detected during the first observing night at a significance level of $3.1 \sigma$. As we show below in Sect. 4, this star has a high kinematical membership probability in the open cluster Hogg 22.

\subsection{2. $H D 157857$}

Spectropolarimetric observations of this star have been conducted for the first time. Our measurements on the second observing night carried out on hydrogen lines resulted in a field detection at a significance level of $3.7 \sigma$. Schilbach \& Roeser (2008) retraced the orbit of HD 157857 in the Galactic potential and concluded that it was ejected from the open cluster NGC 6611 about $3.8 \mathrm{Myr}$ ago with a peculiar velocity of $113.5 \mathrm{~km} \mathrm{~s}^{-1}$.

\subsection{HD 164794}

All four SOFIN and one HARPS high-resolution spectropolarimetric observations confirm our previous detection of the longitudinal magnetic field in this star. The field appears to be variable with negative and positive extreme values of $-265 \mathrm{G}$ and +242 G. HD 164794 belongs to the young cluster NGC 6530 and according to the Galactic O Star Catalogue (Maíz-Apellániz et al. 2004), has a classification O4 V ((f)), exhibiting weak N III emission and strong He II $\lambda 4686$ absorption. It is a wellknown non-thermal radio emitter and, according to van Loo et al. (2006), the most likely mechanism is synchrotron emission from colliding winds, implying that all $\mathrm{O}$ stars with non-thermal radio emission should be members of binary or multiple systems. Hints of a wind-wind interaction were indeed detected in the X-ray domain (Rauw et al. 2002). A long-term study of its binary nature and spectrum variability has been recently presented by Rauw et al. (2012b) who derived an orbital solution and an orbital period of $8.6 \mathrm{yr}$.

\subsection{4. $H D 191612$}

Our SOFIN spectropolarimetric observations of this star have already been discussed in Sect. 2.2. HD 191612 is currently one of the best studied targets in the optical and X-ray domains among the five currently known Galactic Of?p stars. Due to the above mentioned discrepancies in the magnetic field measurements around the positive field extremum, additional magnetic field observations are necessary for proper magnetic field geometry modelling.

\section{Discussion}

Whereas magnetic fields are ubiquitous in low mass stars due to their convective envelopes, and found in $10 . . .20 \%$ of all intermediate mass stars, they were unexpected in $\mathrm{O}$ stars until just a few years ago. The first $\mathrm{O}$ star for which a magnetic field was detected is the spectroscopically variable star $\theta^{1}$ Ori $\mathrm{C}$ (Donati et al. 2002). However, with ever more magnetic $\mathrm{O}$ stars being identified in recent years (e.g., Hubrig et al. 2011b; Martins et al. 2012), it appears likely that the as yet small number of magnetic $\mathrm{O}$ stars is just a consequence of an observational bias, due to the low number of spectral lines in these objects. Indeed, there may be more reasons to find magnetic fields in $\mathrm{O}$ stars than in A and B stars. Fossil fields might appear with similar frequencies on both groups. If close binary effects as mass transfer or stellar merger would produce large scale stable surface fields in O stars (Ferrario et al. 2009; Langer 2012), then about one quarter of them would be expected to be magnetic due to their very high close binary fraction (Sana et al. 2012). Furthermore, their proximity to the Eddington limit produces convection in the envelopes of $\mathrm{O}$ type stars, which could also produce surface magnetic fields (Cantiello et al. 2009).

With the present work we gradually increase the sample of massive stars with determined magnetic field strengths. It is the aim of our survey to provide new clues about the origin and detectability of the magnetic fields of massive stars. Bagnulo et al. (2012) used the ESO FORS 1 pipeline to reduce the full content of the FORS 1 archive. They claim that very small instrument flexures, negligible in most of the instrument applications, may be responsible for some spurious magnetic field detections. We note, however, that out of the five Of?p stars, two were detected to be magnetic for the first time by us (Hubrig et al. 2008, 2011b). The excellent agreement of our FORS 1 and FORS 2 data with ESPaDOnS observations by Wade et al. (2012b) of HD 148937 can be seen in Fig. 6. Bagnulo et al. (2012) could not detect the magnetic field in 9 Sgr (=HD 164794), while our high-resolution observations of this star with both SOFIN and HARPS fully confirm our earlier result (Hubrig et al. 2008). Also, high-resolution observations with ESPaDOnS (Petit et al. 2013) showed a weak magnetic field in 40 Tau (=HD 25558), which was already detected by Hubrig et al. (2009b), but not found by Bagnulo et al. (2012) from the same data. Overall, it is not clear why Bagnulo et al. (2012) are not able to find magnetic-fields found by other groups with FORS $1 / 2$ and other instruments. These are only a few examples that show the need for confirmations of FORS 1 magnetic-field measurements with high spectral resolution polarimeters. In the following, we discuss the role of magnetic stars in star clusters, magnetic runaway stars, and indirect magnetic field indicators in massive stars.

\subsection{Magnetic stars in young clusters}

Of the 30 massive stars analysed in this paper, eleven stars have been found to be related to open clusters of different ages. The data on the cluster membership of these probable cluster O-type stars are presented in Table 4. As database for the compilation of Table 4, we used the All-sky Compiled Catalogue of 2.5 million stars (ASCC-2.5, 3rd version) of Kharchenko \& Roeser (2009). The kinematic and photometric probabilities for cluster membership presented in Cols. 3 and 4 were calculated according to the procedures described by Kharchenko et al. (2004). For calculating the kinematic membership probability, only proper motion information was used. Since the star HD 92206c in the open cluster NGC 3324 belongs to the triple system HD 92206abc, we present the proper motions of all triple system members in the same table. Largely deviating proper motions of the cluster member HD 92206b in comparison to the average cluster proper motions are very likely caused by the orbital motion in the pair HD 92206ab. Interestingly, not only the cluster member HD 92206c shows the presence of a magnetic field. According to Hubrig et al. (2012b), also another cluster member, the supergiant HD 92207, possesses a magnetic field of the order of a few hundred Gauss. Clearly, the cluster NGC 3324 should be included in future magnetic field surveys of massive stars to better understand the role of the environment on the generation of their magnetic fields.

As we already mentioned in our previous work, according to Schilbach \& Roeser (2008), the star HD 153919 was probably ejected from the cluster NGC 6231 at an age of about 6.5 Myr. At present, HD 153919 is located $4.2^{\circ}$ from the centre of 
Table 4. Probable members in open clusters.

\begin{tabular}{|c|c|c|c|c|c|c|c|c|}
\hline $\begin{array}{l}\text { Object } \\
\text { name }\end{array}$ & $\begin{array}{l}\text { ASCC } \\
\text { number }\end{array}$ & $\begin{array}{l}P_{\text {kin }} \\
{[\%]}\end{array}$ & $\begin{array}{l}P_{\text {phot }} \\
{[\%]}\end{array}$ & Cluster & $\begin{array}{l}\text { Dist } \\
{[\mathrm{pc}]}\end{array}$ & $\begin{array}{l}\log t \\
{[\mathrm{yr}]}\end{array}$ & $\begin{array}{c}\left(\mu_{X}, \mu_{Y}, \sigma_{\mu}\right)^{\text {star }} \\
{[\mathrm{mas} / \mathrm{yr}]}\end{array}$ & $\begin{array}{c}\left(\mu_{X}, \mu_{Y}, \sigma_{\mu}\right)^{\mathrm{cl}} \\
{[\mathrm{mas} / \mathrm{yr}]}\end{array}$ \\
\hline${ }^{*}$ HD 47839 & 1021435 & 62 & 100 & NGC 2264 & 660 & 6.81 & $-3.84,-2.50,0.94$ & $-2.70,-3.50,0.25$ \\
\hline HD 92206a & 2231158 & 80 & 96 & NGC 3324 & 2301 & 6.72 & $-10.32, \quad 6.76,4.05$ & $-7.81, \quad 3.77,0.64$ \\
\hline HD 92206b & 2231162 & 0 & 12 & NGC 3324 & 2301 & 6.72 & $49.61, \quad 0.86,3.70$ & $-7.81, \quad 3.77,0.64$ \\
\hline${ }^{*} \mathrm{HD} 92206 \mathrm{c}$ & 2231147 & 90 & 97 & NGC 3324 & 2301 & 6.72 & $-8.87, \quad 4.57,1.90$ & $-7.81, \quad 3.77,0.64$ \\
\hline HD 93026 & 2232306 & 86 & 88 & Bochum 10 & 2027 & 6.93 & $-3.74, \quad 3.69,4.23$ & $-6.61, \quad 2.56,0.62$ \\
\hline CPD-58 2611 & 2232406 & 96 & 100 & Trumpler 14 & 2753 & 6.67 & $-3.09, \quad 4.44,2.66$ & $-3.91, \quad 3.65,0.60$ \\
\hline *HD 328856 & 2074567 & 22 & 100 & Hogg 22 & 1297 & 6.70 & $0.78,-1.37,1.26$ & $-0.84,-4.39,0.43$ \\
\hline${ }^{*} \mathrm{CPD}-468221$ & 2074573 & 97 & 1 & Hogg 22 & 1297 & 6.70 & $-0.45,-5.19,1.32$ & $-0.84,-4.39,0.43$ \\
\hline HD 150958 & 2074576 & 93 & 84 & Hogg 22 & 1297 & 6.70 & $-0.95,-3.67,1.20$ & $-0.84,-4.39,0.43$ \\
\hline HD 153919 & 1877055 & 0 & 100 & NGC 6281 & 494 & 8.51 & $1.42, \quad 4.84,1.04$ & $-2.96,-3.75,0.23$ \\
\hline HD 156041 & 1878768 & 98 & 63 & Bochum 13 & 1077 & 7.08 & $0.03,-0.74,1.70$ & $-0.28,-1.20,0.30$ \\
\hline HD 156154 & 1878846 & 82 & 100 & Bochum 13 & 1077 & 7.08 & $-0.88,-2.53,1.64$ & $-0.28,-1.20,0.30$ \\
\hline *HD 164794 & 1593528 & 69 & 100 & NGC 6530 & 1322 & 6.67 & $1.92,-0.40,1.09$ & $2.01,-1.81,0.51$ \\
\hline
\end{tabular}

Notes. For each star, we give in the first two columns the object name and the corresponding ASCC catalogue number. The kinematic and photometric probabilities for cluster membership presented in Cols. 3 and 4 were calculated according to the procedures described by Kharchenko et al. (2004). The cluster names, the distances, and the ages are presented in the next three columns. The proper motions and their errors for stars and clusters are given in the last columns. All cluster data are taken from Kharchenko et al. (2005a). Stars with detected magnetic fields are marked with an asterisk in the first column.

NGC 6231. Considering the proper motions of HD 153919 given by Kharchenko \& Roeser $\left(2009 ; \mu_{\alpha}=+1.42 \pm 1.17 \mathrm{mas} / \mathrm{yr}, \mu_{\delta}=\right.$ $+4.84 \pm 0.87$ mas/yr) and those of the cluster by Kharchenko et al. $(2005 \mathrm{a} ;-0.39 \pm 0.52 \mathrm{mas} / \mathrm{yr},-1.99 \pm 0.38 \mathrm{mas} / \mathrm{yr})$, then $2.15 \mathrm{Myr}$ ago the star was at $0.55 \pm 0.95^{\circ}$ from the cluster centre.

Among the stars presented in Table 4, only five stars show the presence of magnetic fields, HD 47839, HD 92206c, HD 328856, CPD-468221, and HD 164794. Two of them, HD 328856 and CPD-468221, belong to the open cluster Hogg 22, which appears as a second promising cluster for investigating the magnetic nature of cluster members. Although we deal here with small number statistics, we cannot refrain from mentioning that these five stars with detected magnetic fields belong to the youngest clusters in our small sample.

\subsection{Magnetic massive runaway stars}

Magnetic fields are detected in stars of spectral types, with different kinematical status, and at different evolutionary stages. Clearly, the detection of magnetic fields in all five Galactic Of?p stars implies a tight relation between the spectral characteristics of the Of?p star group and the presence of a magnetic field. Remarkably, apart from the Of?p star NGC 1624-2, no other Of?p star belongs to an open cluster or association. The analysis of the stellar content of the cluster NGC 1624 by Jose et al. (2011) indicated that NGC 1624-2 is the main ionising source of the region, and the maximum post-main-sequence age of the cluster is estimated as $\sim 4 \mathrm{Myr}$.

Furthermore, recent studies of the evolutionary state of several magnetic massive stars indicate that some of them evolved significantly or have the status of a runaway star (e.g., Martins et al. 2010; Hubrig et al. 2011b,d). In the literature, the runaway status or candidate runaway status (i.e., if stars possess high space velocities determined through proper motion and/or radial velocity measurements) is assigned to several magnetic massive stars, such as $\zeta$ Oph (Blaauw 1952), HD 93521 (Gies 1987), HD 157857 (Schilbach \& Roeser 2008), HD 57682 (Comeron et al. 1998), and the three Of?p stars HD 108, HD 148937 , HD 191612 (Hubrig et al. 2011d). It is known that some of the massive stars located far from star clusters and star-forming regions are runaways. They were likely formed in embedded clusters and then ejected into the field because of dynamical fewbody interactions or binary-supernova explosions (Eldridge et al. 2011). Although the group of field O stars whose runaway status is difficult to prove does exist, the recent study of Gvaramadze et al. (2012) showed no significant evidence in support of the in-situ proposal for the origin of isolated massive stars.

In some cases, even if the star is considered as an open cluster member, a careful study of their kinematic characteristics indicates that it is not fully co-moving with the other cluster members. As an example, the space motion of the magnetic O-type $\operatorname{star} \theta^{1}$ Ori C was studied by van Altena et al. (1988), who reported that $\theta^{1}$ Ori $\mathrm{C}$ is moving at $4.8 \pm 0.5 \mathrm{~km} \mathrm{~s}^{-1}$ towards position angle $142^{\circ}$ and that this velocity is significantly larger than the dispersion value of $1.5 \pm 0.5 \mathrm{~km} \mathrm{~s}^{-1}$ found for the other cluster members. The results of the radial velocity study of Stahl et al. (2008) indicate that this star is moving rapidly away from the Orion Molecular Cloud and its host cluster. Another example is the kinematical status of the magnetic O-type star HD 328856 , which is on the basis of the photometric membership probability a member of the compact open cluster Hogg 22 in the Ara region at an age of $5 \mathrm{Myr}$ and a distance of about $1300 \mathrm{pc}$. However, its proper motions indicate that HD 328856 is not fully co-moving with the other cluster members, deviating from the cluster mean proper motion by $\sim 2 \sigma$ (Hubrig et al. 2011b).

Among our studied runaway stars, HD 93521 and $\zeta$ Oph are magnetic fast rotators with $\beta$ Cep-type pulsations. These stars are thus particularly interesting to calibrate stellar rotation models including magnetic fields in the hot upper part of the Hertzsprung-Russell diagram. Variability linked to stellar pulsation has never been searched for or found in any of our other sample targets. In fact, there are only a few O-type stars for which convincing evidence of non-radial pulsations with periods of a few hours has been detected from ground-based observations. Besides HD 93521 and $\zeta$ Oph, there are $\xi$ Per and $\lambda$ Cep (de Jong et al. 1999). For such stars, the photometric amplitudes are below mmag level, which are very difficult to detect from the ground. It may explain why only few pulsating O-type stars have been identified so far and mostly spectroscopically. Recently, the very high-precision photometry of the CoRoT satellite led to the detection of stellar pulsation in three other O-type objects that 
belong to the young open cluster NGC 2244 inside the Rosette nebula and to the surrounding association Mon OB2. $\beta$ Cep-like pulsation modes have been found in HD 46202 (Briquet et al. 2011) and in HD 47129 (Mahy et al. 2011) while unexpected modes of stochastic nature have been detected in HD 46149 (Degroote et al. 2010). Efforts to discover magnetic massive pulsators are worthwhile since their study provides a unique opportunity to probe the impact of a magnetic field on the physics of mixing inside stellar interiors.

\subsection{Indirect B-field indicators}

One of the major characteristics of magnetic Ap and Bp stars is the spectral variability due to the presence of chemical spots showing a certain symmetry with respect to the magnetic field geometry. Although spectral variability is present in all discovered magnetic massive stars, the interpretation of line profile variations seems not to be as simple as for Ap and Bp stars: the behaviour of line profiles of different ions of a given element does not clearly correspond to the abundance variations. Synthetic spectra calculations of the line profile variations in massive stars are furthermore more troublesome since different spectral lines are to a different degree sensitive to the stellar wind and to non-LTE effects. In any case, a detection of line profile variations can certainly be used as an indirect indicator for the possible presence of a magnetic field. Another aspect that could hint at the presence of a magnetic field in massive stars is the fact that a number of magnetic OB-type stars show nitrogen enrichment (e.g. Morel et al. 2008).

It is not clear yet how to use the X-ray emission as an indirect indicator for the presence of magnetic fields. Anomalous X-ray emission can be expected from a massive star with a magnetic field strong enough to regulate the stellar wind motion. The relative importance of magnetic fields in gases is described by the plasma- $\beta_{\mathrm{p}}$ parameter with $\beta_{\mathrm{p}}=8 \pi p / B^{2}$, where $p$ is the gas pressure. The gas is magnetically dominated when $\beta_{\mathrm{p}}<1$. For supersonic flows, such as stellar winds, the ram pressure exceeds the gas pressure and the dynamical importance of a magnetic field is defined by the ratio of wind kinetic to magnetic energy density: when this ratio is less than one, the magnetic field dominates the wind bulk motion.

Oskinova et al. (2011) investigated the X-ray emission and wind properties among the complete sample of known magnetic early B-type stars with available X-ray observations. The authors have found that the X-ray emission from magnetic B-type stars is diverse. While some stars display hard variable X-rays, others are rather soft sources. The X-ray luminosities differ among otherwise similar Bp stars by more than two orders of magnitude. Among magnetic O-type stars, the situation is similar. While some of them are bright, hard, variable X-ray emission as predicted by the magnetically confined wind-shock scenario (e.g. Schulz et al. 2000), others are relatively soft and faint X-ray sources (Nazé et al. 2010).

Thus, the analysis of X-ray observations of magnetic OB stars shows that some of them are lacking strong and hard $\mathrm{X}$-ray emission, or even are X-ray dark. Therefore, while a strong hard X-ray emission serves as a good indicator for the potential presence of a strong stellar magnetic field, X-ray strong stars represent only a sub-class of all magnetic massive stars.

\section{Outlook}

What is the origin of the magnetic fields in massive stars? It is quite possible that massive O-type stars behave similar to slowly rotating, chemically peculiar, magnetic Ap and Bp stars, which amount for 10 to $20 \%$ of main-sequence A and B stars. It was suggested that their magnetic fields are inherited from their parent molecular clouds (Moss 2003). Alternatively, from analysing possible paths for the formation of Ap and Bp stars based on modern theories for the evolution of single and binary stars, Tutukov \& Fedorova (2010) suggested that merging of close binaries is their main formation channel.

Indeed, the evolution of a high fraction of massive stars is affected by environmental effects. Sana et al. (2012) find two thirds of all Galactic O stars to be members of close binary systems. Mass transfer or stellar merger may rejuvenate the mass gaining star while strong differential rotation may cause the creation of a magnetic field (Langer 2012). In dense star clusters, close multibody interactions may cause single and binary stars to be scattered out of the star cluster (e.g. Leonard \& Duncan 1990).

Clearly, these various ideas bear significantly different predictions for the appearance of magnetic main sequence stars inside and outside of star clusters. This means that these predictions are well testable, and we are confident that the question of the origin of the magnetic fields in massive main sequence stars will be answered in the near future. As described in Sect. 4, first trends are emerging from the scarce data at hand. However, at the current stage, we clearly need more observations before firm conclusions can be drawn.

Acknowledgements. This research made use of the SIMBAD database, operated at CDS, Strasbourg, France. The Nordic Optical Telescope is operated on the island of La Palma jointly by Denmark, Finland, Iceland, Norway, and Sweden, in the Spanish Observatorio del Roque de los Muchachos of the Instituto de Astrofisica de Canarias. We would like to thank G. Lo Curto for his help with the HARPS data reduction, and V. Gvaramadze for stimulating discussions. S.H. and J.F.G. acknowledge support by the Deutsche Forschungsgemeinschaft (Hu532/17-1)

\section{References}

Babel, J., \& Montmerle, Th. 1997, ApJ, 485, 29

Bagnulo, S., Landstreet, J. D., Fossati, L., \& Kochukhov, O. 2012, A\&A, 538, A129

Benaglia, P., Cappa, C. E., \& Koribalski, B. S. 2001, A\&A, 372, 952

Blaauw, A. 1952, Bull. Astron. Inst. Netherlands, 11, 414

Briquet, M., Aerts, C., Lüftinger, T., et al. 2004, A\&A, 413, 273

Briquet, M., Aerts, C., Baglin, A., et al. 2011, A\&A, 527, A112

Brott, I., Evans, C. J., Hunter, I., et al. 2011, A\&A, 530, A116

Cantiello, M., Langer, N., Brott, I., et al. 2009, A\&A, 499, 279

Cappa, C., Niemela, V. S., Amorín, R., \& Vasquez, J. 2008, A\&A, 477, 173

Chlebowski, T. 1989, ApJ, 342, 1091

Comeron, F., Torra, J., \& Gomez, A. E. 1998, A\&A, 330, 975

Degroote, P., Briquet, M., Auvergne, M., et al. 2010, A\&A, 519, A38

de Jong, J. A., Henrichs, H. F., Schrijvers, C., et al. 1999, A\&A, 345, 172

Donati, J.-F., Semel, M., Carter, B. D., et al. 1997, MNRAS, 291, 658

Donati, J.-F., Babel, J., Harries, T. J., et al. 2002, MNRAS, 333, 55

Donati, J.-F., Howarth, I. D., Bouret, J.-C., et al. 2006, MNRAS, 365, L6

Eldridge, J. J., Langer, N., Tout, C. A., et al. 2011, MNRAS, 414, 3501

Ferrario, L., Pringle, J. E., Tout, C. A., \& Wickramasinghe, D. T. 2009, MNRAS, 400, L1

Fürst, F., Kreykenbohm, I., Pottschmidt, K., et al. 2010, A\&A, 519, A37

Gies, D. R. 1987, ApJS, 64, 545

Gies, D. R., Mason, B. D., Hartkopf, W. I., et al. 1993, AJ, 106, 2072

Gvaramadze, V. V., Weidner, C., Kroupa, P., \& Pflamm-Altenburg, J. 2012, MNRAS, 424, 3037

Howarth, I. D., \& Reid, A. H. N. 1993, A\&A, 279, 148

Howarth, I. D., Walborn, N. R., Lennon, D. J., et al. 2007, MNRAS, 381, 433

Hubrig, S., Kurtz, D. W., Bagnulo, S., et al. 2004a, A\&A, 415, 661

Hubrig, S., Szeifert, T., Schöller, M., et al. 2004b, A\&A, 415, 685

Hubrig, S., Briquet, M., Schöller, M., et al. 2006, MNRAS, 369, L61

Hubrig, S., Schöller, M., Schnerr, R. S., et al. 2008, A\&A, 490, 793

Hubrig, S., Stelzer, B., Schöller, M., et al. 2009a, A\&A, 502, 283 
S. Hubrig et al.: Exploring the origin of magnetic fields in massive stars. II.

Hubrig, S., Briquet, M., De Cat, P., et al. 2009b, Astron. Nachr., 330, 317 Hubrig, S., Ilyin, I., \& Schöller, M. 2010, AN, 331, 781

Hubrig, S., Schöller, M., Ilyin, I., et al., 2011a, ApJ, 726, L5

Hubrig, S., Schöller, M., Kharchenko, N. V., et al. 2011b, A\&A, 528, A151

Hubrig, S., Oskinova, L. M., Schöller, M. 2011c, AN, 332, 147

Hubrig, S., Kharchenko, N. V., \& Schöller, M. 2011d, AN, 332, 65

Hubrig, S., Kholtygin, A., Schöller, M., et al. 2012a, IBVS, 6019, 1

Hubrig, S., Schöller, M., Kholtygin, A. F., et al. 2012b, A\&A, 546, L6

Ignace, R., Oskinova, L., \& Massa, D. 2013, MNRAS, 429, 516

Ilyin, I. 2000, Ph.D. Thesis, University of Oulu, Finland

Ilyin, I. 2012, Astron. Nachr., 333, 213

Jose, J., Pandey, A. K., Ogura, K., et al. 2011, MNRAS, 411, 2530

Kambe, E., Ando, H., \& Hirata, R. 1993, A\&A, 273, 4

Kambe, E., Hirata, R., Ando, H., et al. 1997, ApJ, 481, 406

Kaper, L., Henrichs, H. F., Nichols, J. S., et al. 1996, A\&AS, 116, 257

Kaper, L., Henrichs, H. F., Fullerton, A. W., et al. 1997, A\&A, 327, 281

Kharchenko, N. V., \& Roeser, S. 2009, All-sky Compiled Catalogue of 2.5 million stars

Kharchenko, N. V., Piskunov, A. E., Röser, S., et al. 2004, AN, 325, 740

Kharchenko, N. V., Piskunov, A. E., Röser, S., et al. 2005, A\&A, 438, 1163

Kholtygin, A. F., Monin, D. N., Surkov, A. E., \& Fabrika, S. N. 2003, Astron. Lett., 29, 175

Koenigsberger, G., Moreno, E., \& Harrington, D. M. 2012, A\&A, 539, A84

Kreykenbohm, I., Wilms, J., Kretschmar, P., et al. 2008, A\&A, 492, 511

Langer, N. 2012, ARA\&A, 50, 107

Leonard, P. J. T., \& Duncan, M. J. 1990, AJ, 99, 608

Levato, H., Morrell, N., Garcia, B., \& Malaroda, S. 1988, ApJS, 68, 319

Mahy, L., Gosset, E., Baudin, F., et al. 2011, A\&A, 525, A101

Maíz-Apellániz, J., Walborn, N. R., Galué, H. Á., \& Wei, L. H. 2004, ApJS, 151, 103

Markova, N., Puls, J., Repolust, T., \& Markov, H. 2004, A\&A, 413, 693

Martins, F., Donati, J.-F., Marcolino, W. L. F., et al. 2010, MNRAS, 407, 1423

Martins, F., Escolano, C., Wade, G. A., et al. 2012, A\&A, 538, A29

Mathys. G. 1993, in Peculiar versus Normal Phenomena in A-type and Related Stars, eds. M. M. Dworetsky, F. Castelli, \& R. Faraggiana (San Francisco: ASP), ASP Conf. Ser., 44, 232

Mathys, G. 1995a, A\&A, 293, 733

Mathys, G. 1995b, A\&A, 293, 746

Mathys, G., \& Hubrig, S. 1995, A\&A, 293, 810

Mayor, M., Pepe, F., Queloz, D., et al. 2003, The ESO Messenger, 114, 20

Morel, T., Hubrig, S., \& Briquet, M. 2008, A\&A, 481, 453

Moss, D. 2003, A\&A, 403, 693

Nazé, Y. 2009, A\&A, 506, 1055

Nazé, Y., Walborn, N. R., Rauw, G., et al. 2008, AJ, 135, 1946

Nazé, Y., ud-Doula, A., Spano, M., et al. 2010, A\&A, 520, A59

Nazé, Y., Broos, P. S., Oskinova, L., et al. 2011, ApJS, 194, 7
Oskinova, L. M. 2005, MNRAS, 361, 679

Oskinova, L. M., Clarke, D., Pollock, A. M. T. 2001, A\&A, 378, L21

Oskinova, L. M., Todt, H., Ignace, R., et al. 2011, MNRAS, 416, 1456

Oskinova, L. M., Feldmeier, A., \& Kretschmar, P. 2012, MNRAS, 421, 2820

Petit, V., Owocki, S. P., Wade, G. A., et al. 2013, MNRAS, 429, 398

Pollmann, E. 2012, IBVS, 6034, 1

Potter, A. T., Chitre, S. M., \& Tout, C. A. 2012, MNRAS, 424, 2358

Prinja, R. K., Massa, D., Howarth, I. D., \& Fullerton, A. W. 1998, MNRAS, 301, 926

Ramirez S.V., Rebull L., Stauffer J., et al. 2004, AJ, 127, 2659

Rauw, G., Blomme, R., Waldron, W. L., et al. 2002, A\&A, 394, 993

Rauw, G., De Becker, M., van Winckel, H., et al. 2008, A\&A, 487, 659

Rauw, G., Morel, T., \& Palate, M. 2012a, A\&A, 546, A77

Rauw, G., Sana, H., Spano, M., et al. 2012b, A\&A, 542, A95

Sana, H., de Mink, S. E., de Koter, A, et al. 2012. Science, 337, 444

Schilbach, E., \& Röser, S. 2008, A\&A, 489, 105

Schulz, N. S., Canizares, C. R., Huenemoerder, D., \& Lee, J. C. 2000, ApJ, 545, L135

Snik, F., Kochukhov, O., Piskunov, N., et al. 2011, in Solar Polarization 6, eds. J. R. Kuhn, D. M. Harrington, H. Lin, S. V. Berdyugina, J. Trujillo-Bueno, S. L. Keil, \& T. Rimmele (San Francisco: ASP), ASP Conf. Ser., 437, 237

Stahl, O., Wade, G., Petit, V., et al. 2008, A\&A, 487, 323

Sung, H., Bessell, M. S., Chun, M.-Y., et al. 2008, AJ, 135, 441

Teixeira, P. S., Lada, C. J., Marengo, M., \& Lada, E. A. 2012, A\&A, 540, A83

Townsley, L. K., Broos, P. S., Corcoran, M. F., et al. 2011, ApJS, 194, 1

Tuominen, I., Ilyin, I., \& Petrov, P. 1999, in Astrophysics with the NOT, eds. H. Karttunen, \& V. Piirola, University of Turku, Tuorla Observatory, 47

Tutukov, A. V., \& Fedorova, A. V. 2010, Astron. Rep., 54, 808

van Altena, W. F., Lee, J. T., Lee, J.-F., et al. 1988, AJ, 95, 1744

van Loo, S., Runacres, M. C., \& Blomme, R. 2006, A\&A, 452, 1011

Wade, G. A., Howarth, I. D., Townsend, R. H. D., et al. 2011, MNRAS, 416, 3160

Wade, G. A., Maíz Apellániz, J., Martins, F., et al. 2012a, MNRAS, 425, 1278

Wade, G. A., Grunhut, G., Gräfener, G., et al. 2012b, MNRAS, 419, 2459

Walborn, N. R. 1973, AJ, 78, 1067

Walborn, N. R. 1982, AJ, 87, 1300

Walborn, N. R. 2006, in The Ultraviolet Universe: Stars from Birth to Death, 26th meeting of the IAU, Joint Discussion 4, 16-17 August 2006, Prague, Czech Republic, JD04, \#19

Walborn, N. R., \& Panek, R. J. 1984, ApJ, 286, 718

Walborn, N. R., Nichols, J. S., \& Waldron, W. L. 2009, ApJ, 703, 633

Walborn, N. R., Sota, A., Maíz Apellániz, J., et al. 2010, ApJ, 711, L143

Waldron, W. L. 2005, in X-ray Diagnostics of Astrophysical Plasmas: Theory, Experiment, and Observation, ed. R. Smith, AIPC, 774, 353

Waldron, W. L., \& Cassinelli, J. P. 2007, ApJ, 668, 456

Walker, G. A. H., Kuschnig, R., Matthews, J. M., et al. 2005, ApJ, 623, 145 